\title{
PHOTOMETRIC ESTIMATES OF REDSHIFTS AND DISTANCE MODULI FOR TYPE Ia SUPERNOVAE
}

\author{
Richard Kessler ${ }^{1,2}$, David Cinabro $^{3}$, Bruce Bassett $^{4,5}$, Benjamin Dilday $^{6}$, Joshua A. Frieman $^{1,2,7}$, \\ Peter M. Garnavich ${ }^{8}$, Saurabh Jha ${ }^{6}$, John Marriner ${ }^{7}$, Robert C. Nichol ${ }^{9}$, Masao Sako $^{10}$, Mathew Smith $^{4}$, \\ Joseph P. Bernstein ${ }^{11}$, Dmitry Bizyaev ${ }^{12}$, Ariel Goobar ${ }^{13,14}$, STePhen Kuhlmann ${ }^{11}$, Donald P. Schneider ${ }^{15}$, \\ and Maximilian Stritzinger ${ }^{16,17}$ \\ ${ }^{1}$ Department of Astronomy and Astrophysics, The University of Chicago, 5640 South Ellis Avenue, Chicago, IL 60637, USA; kessler@kicp.uchicago.edu \\ ${ }^{2}$ Kavli Institute for Cosmological Physics, The University of Chicago, 5640 South Ellis Avenue Chicago, IL 60637, USA \\ ${ }^{3}$ Department of Physics and Astronomy, Wayne State University, Detroit, MI 48202, USA \\ ${ }^{4}$ Department of Mathematics and Applied Mathematics, University of Cape Town, Rondebosch 7701, South Africa \\ ${ }^{5}$ South African Astronomical Observatory, P.O. Box 9, Observatory 7935, South Africa \\ ${ }^{6}$ Department of Physics and Astronomy, Rutgers University, 136 Frelinghuysen Road, Piscataway, NJ 08854, USA \\ ${ }^{7}$ Center for Particle Astrophysics, Fermi National Accelerator Laboratory, P.O. Box 500, Batavia, IL 60510, USA \\ ${ }^{8}$ University of Notre Dame, 225 Nieuwland Science, Notre Dame, IN 46556-5670, USA \\ ${ }^{9}$ Institute of Cosmology and Gravitation, Mercantile House, Hampshire Terrace, University of Portsmouth, Portsmouth PO1 2EG, UK \\ ${ }^{10}$ Department of Physics and Astronomy, University of Pennsylvania, 203 South 33rd Street, Philadelphia, PA 19104, USA \\ ${ }^{11}$ Argonne National Laboratory, 9700 S. Cass Avenue, Lemont, IL 60437, USA \\ ${ }_{12}$ Apache Point Observatory, P.O. Box 59, Sunspot, NM 88349, USA \\ ${ }^{13}$ Department of Physics, Stockholm University, Albanova University Center, SE-106 91 Stockholm, Sweden \\ ${ }^{14}$ The Oskar Klein Centre for Cosmoparticle Physics, Department of Physics, AlbaNova, Stockholm University, SE-106 91 Stockholm, Sweden \\ ${ }^{15}$ Department of Astronomy and Astrophysics, The Pennsylvania State University, 525 Davey Laboratory, University Park, PA 16802, USA \\ ${ }^{16}$ Las Campanas Observatory, Carnegie Observatories, Casilla 601, La Serena, Chile \\ ${ }^{17}$ Dark Cosmology Centre, Niels Bohr Institute, University of Copenhagen, Juliane Maries Vej 30, 2100 Copenhagen $\emptyset$, Denmark \\ Received 2010 January 3; accepted 2010 April 20; published 2010 June 7
}

\begin{abstract}
Large planned photometric surveys will discover hundreds of thousands of supernovae (SNe), outstripping the resources available for spectroscopic follow-up and necessitating the development of purely photometric methods to exploit these events for cosmological study. We present a light curve fitting technique for type Ia supernova (SN Ia) photometric redshift (photo- $z$ ) estimation in which the redshift is determined simultaneously with the other fit parameters. We implement this "LCFIT $+Z$ " technique within the frameworks of the MLCS2K2 and SALTII light curve fit methods and determine the precision on the redshift and distance modulus. This method is applied to a spectroscopically confirmed sample of 296 SNe Ia from the Sloan Digital Sky Survey-II (SDSS-II) SN Survey and 37 publicly available SNe Ia from the Supernova Legacy Survey (SNLS). We have also applied the method to a large suite of realistic simulated light curves for existing and planned surveys, including the SDSS, SNLS, and the Large Synoptic Survey Telescope. When intrinsic SN color fluctuations are included, the photo- $z$ precision for the simulation is consistent with that in the data. Finally, we compare the LCFIT $+Z$ photo- $z$ precision with previous results using color-based SN photo- $z$ estimates.
\end{abstract}

Key words: methods: data analysis - supernovae: general - techniques: photometric

Online-only material: color figures

\section{INTRODUCTION}

To investigate the expansion history of the universe, increasingly large samples of high-quality type Ia supernova (SN Ia) light curves are being used to measure luminosity distances as a function of redshift (the SN Ia Hubble diagram). Expected SN Ia samples will be in the thousands for the Dark Energy Survey (DES; Bernstein et al. 2009) and in the hundreds of thousands for the surveys to be carried out by the Panoramic Survey Telescope and Rapid Response System (Pan-STARRS) ${ }^{18}$ and by the Large Synoptic Survey Telescope (LSST; Ivezić et al. 2008; LSST Science Collaborations 2009). For the latter two, only a small fraction of the SNe will have spectroscopically determined redshifts from SN or host-galaxy spectra for the foreseeable future. To make use of these large SN samples, the redshifts will have to be determined photometrically using both the SN light curves and the host-galaxy photometric observables.

Methods for estimating galaxy photo-z's have been developed over many years (for a review, see, e.g., Abdalla et al.

18 http://pan-starrs.ifa.hawaii.edu/public
2008). They generally fall into two categories: (1) an empirical approach, in which one translates observed colors, magnitudes, or other photometric observables into a redshift estimate, training the algorithm on a subset of galaxies with spectroscopic redshifts and (2) template fitting, in which observed colors are matched to redshifted template galaxy spectral energy distributions until the best match for galaxy redshift and type is obtained. The development of photo- $z$ methods using SN data is more recent. Some have followed and adapted the empirical approach to galaxy photo-z estimation, e.g., using observed SN colors near the epoch of peak brightness to estimate the redshift (Wang 2007; Wang et al. 2007) or early-epoch colors to select SNe in particular redshift regions (Dahlen \& Goobar 2002).

In this paper, we present and describe a method of SN Ia photo- $z$ estimation that is analogous to the template fitting method for galaxy photo-z's. In models used to fit SN Ia light curves, one typically uses a spectroscopically determined redshift, and the fit parameters are usually taken to be the epoch of peak brightness, the light curve shape or stretch, a color or dust extinction estimate, and the distance modulus. In our approach, we extend the usual methods of fitting light curves to include the 
redshift as a fifth fit parameter. We apply this "LCFIT+Z" method to determine photo-z's for the spectroscopically confirmed Sloan Digital Sky Survey-II (SDSS-II; Frieman et al. 2008; Kessler et al. 2009a) and Supernova Legacy Survey (SNLS; Astier et al. 2006, hereafter A06) SNe, and compare the resulting photo- $z$ precision to that obtained from simulated samples. Once we have verified the reliability of the simulations by comparison with the data, we apply the LCFIT $+Z$ method to simulated LSST SN observations. In all cases, we use both MLCS2K2 (Jha et al. 2007; Kessler et al. 2009a) and SALTII (Guy et al. 2007) light curve fitting models.

Variants of the light curve fit approach to SN photo- $z$ 's have been used before, by both the SNLS (Sullivan et al. 2006) and SDSS (Sako et al. 2008) surveys, to select SN Ia candidates for spectroscopic follow-up after only a few photometric epochs. Kim \& Miquel (2007) used the sALTII model and a Fisher matrix analysis to estimate uncertainties on photometric redshifts and distances. Using a technique similar to our LCFIT $+Z$ method, Gong et al. (2010) studied LSST simulations, focusing mainly on contamination from non-Ia $\mathrm{SNe}$ and the resulting precision on cosmological parameters. In contrast, we focus here on the precision and bias for the photo- $z$ and distance modulus. We also use more realistic simulations based on the LSST cadence for both the deep and wide surveys and illustrate some differences between these two components of the LSST survey. Our estimates of non-Ia contamination and cosmological precision will be presented in a future work. Recently, PalanqueDelabrouille et al. (2009, hereafter PD09) employed the light curve fit photo- $z$ technique within the SALTII framework. For nearly 300 SNe Ia from the SNLS, they evaluated both the photo$z$ precision and the fraction of catastrophic redshift outliers. The PD09 SN sample is by far the largest to date used to study SN photo- $z$ methods; our SDSS-II sample, at lower redshifts, is of comparable size.

The plan of this paper is as follows. We introduce the spectroscopically confirmed SN data samples (SDSS-II and SNLS) in Section 2. In Section 3, we describe the simulation, and we present the LCFIT $+Z$ method in detail in Section 4 . The photo$z$ precision and fit parameter correlations for the data samples, along with the corresponding results from simulated samples, are presented in Section 5. We use the simulation to make predictions for the LSST survey in Section 6. In Section 7, we make direct comparisons with the color-based photo- $z$ method presented in previous works.

As described in Kessler et al. (2009b), all light curve fitting and simulation software is publicly available in the SNANA package. $^{19}$

\section{THE SDSS-II AND SNLS DATA SAMPLES}

To test the LCFIT $+Z$ method, we use the full three-season sample from the SDSS-II Supernova (SN) Survey (Frieman et al. 2008), and the publicly available sample from the first season of the SNLS (A06). Below we give a brief description of these samples.

The SDSS-II SN Survey used the SDSS camera (Gunn et al. 1998) on the SDSS $2.5 \mathrm{~m}$ telescope (Gunn et al. 2006) at the Apache Point Observatory to search for $\mathrm{SNe}$ in the Fall seasons (September 1 through November 30) of 2005-2007. This survey scanned a region (designated stripe 82) centered on the celestial equator in the Southern Galactic hemisphere

\footnotetext{
19 http://www.sdss.org/supernova/SNANA.html
}

that is 2.5 wide and runs between right ascensions of $20^{\mathrm{h}}$ and $4^{\mathrm{h}}$, covering a total area of $300 \mathrm{deg}^{2}$ with a typical cadence of every four nights per region. Images were obtained in five broad passbands, ugriz (Fukugita et al. 1996), with $55 \mathrm{~s}$ exposures and processed through the PHOTO photometric pipeline (Lupton et al. 2001). Within $24 \mathrm{hr}$ of collecting the data, the images were searched for SN candidates that were selected for spectroscopic follow-up observations in a program involving about a dozen telescopes. The SDSS-II SN Survey discovered and spectroscopically confirmed a total of $\sim 500$ $\mathrm{SNe}$ Ia. A larger sample of photometrically identified but spectroscopically unobserved SNe Ia was also compiled, and host-galaxy redshifts for several hundred of these photometric candidates have been obtained to date. The SDSS-III Survey (Schlegel et al. 2009), as a small part of its early program, is in the process of measuring host-galaxy redshifts for more than 1000 of these photometrically identified SNe Ia. The telescope aperture, focal plane, and exposure time of the SDSS system (York et al. 2000) were ideal for discovering SNe in the previously underexplored redshift range $0.1<z<0.3$. Details of the SDSS-II SN Survey are given in Frieman et al. (2008) and Sako et al. (2008), the procedures for spectroscopic identification and redshift determinations are described in Zheng et al. (2008), and the SN photometry is described in Holtzman et al. (2008). A condensed summary of the SDSS-II survey, SN typing, redshift determination, photometry, and calibration can be found in Kessler et al. (2009a).

The SNLS was a five-year survey covering $4 \mathrm{deg}^{2}$ using the MegaCam imager on the $3.6 \mathrm{~m}$ Canada-France-Hawaii Telescope (CFHT). Images were taken in four bands similar to those used by the SDSS: $g_{M}, r_{M}, i_{M}, z_{M}$, where the subscript $M$ denotes the MegaCam system. The SNLS exposures were $\sim 1 \mathrm{hr}$ in order to discover SNe at redshifts up to $z \sim 1$. The SNLS images were processed in a fashion similar to the SDSSII so that spectroscopic observations could be used to confirm the identities and determine the redshifts of the SN candidates. We use the publicly available sample from their first year of operations that ended 2004 July 15. Detailed information about the SNLS can be found in A06 and references therein.

Since high-quality light curves are needed for the LCFIT $+Z$ method, we apply the following selection requirements to the photometric data for inclusion in our analysis samples: (1) spectroscopic confirmation of type Ia, (2) a measurement with $T_{\text {rest }}<-3$ days, (3) a measurement with $T_{\text {rest }}>+10$ days, (4) measurements in at least three observer-frame filters have signalto-noise ratio $(\mathrm{S} / \mathrm{N})>8$, and (5) the probability corresponding to the fit- $\chi^{2} / N_{\text {dof }}$ (Section 4) is $P_{\chi^{2}}>0.02$. Here, $T_{\text {rest }}$ is the epoch in the SN rest frame relative to peak brightness in the $B$ band, and we note that it depends on the fitted photo- $z$ value. For the SDSS-II, we use only the gri passbands. The number of SNe Ia satisfying these selection requirements is nearly 300 and 40 for the SDSS-II and SNLS, respectively; the exact numbers of SNe depend on the fitting model (MLCS2K2 or SALTII) and will be given in Section 5. The selection criteria above are not based on optimizations, but are instead motivated by the strong correlation between redshift and color. Requiring two colors and at least one measurement in each of three passbands with $\mathrm{S} / \mathrm{N}>8$ explicitly ensures good color measurements. The $T_{\text {rest }}$ requirements $(<-3$ and $>+10$ days) ensure a good determination of the time of peak brightness $\left(t_{0}\right)$; since $\mathrm{SNe}$ become redder after peak light, a mismeasurement of $t_{0}$ translates directly into a mismeasurement of color, and hence redshift. 
Here we provide some additional motivation for the above requirements. Relaxing the $\mathrm{S} / \mathrm{N}$ requirement (No. 4 above) to $\mathrm{S} / \mathrm{N}>5$ results in about $10 \%$ more SNe Ia in the SDSS-II sample, and a $20 \%$ degradation in the photo- $z$ precision. Making a more restrictive cut of $\mathrm{S} / \mathrm{N}>10$ results in a $20 \%$ loss of events and a negligible improvement in the precision. We have therefore chosen $\mathrm{S} / \mathrm{N}>8$ as a reasonable compromise between sample statistics and precision. To motivate the sampling requirements, we have applied the LCFIT $+Z$ fitting method (Section 4) to the SDSS-II sample in which all measurements prior to peak brightness have been rejected; the resulting precision and bias on the fitted $t_{0}$ and photo- $z$ are significantly degraded. The sampling requirements above (Nos. 2 and 3 ) are therefore designed to ensure a good determination of $t_{0}$.

\section{SIMULATIONS}

We use the SNANA simulation code to generate realistic SN Ia light curves that can be analyzed in exactly the same manner as the data. The simulation is used to compare with the data, to compare with previous photo- $z$ studies based on simulations, and to make predictions for LSST. All SN simulations are based on a standard $\Lambda$ CDM cosmology $\left(w=-1, \Omega_{\mathrm{M}}=0.3\right.$, $\Omega_{\Lambda}=0.7$ ), and they are generated and fit using the same light curve model: MLCS2K2 or SALTII. This strategy explicitly assumes that the light curve model is correct and will therefore yield the most optimistic results. As discussed below, the models are adjusted to account for the anomalous Hubble scatter. However, these models have not been adjusted to account for the discrepancies in the ultraviolet region (Kessler et al. 2009a, hereafter K09). Details of the simulation are described in Kessler et al. (2009b) and in Section 6 of K09; here we give a brief overview.

For MLCS2K2, rest-frame model magnitudes are generated from light curve templates and then dimmed by host-galaxy dust extinction. Using SN Ia spectral templates from Hsiao et al. (2007), $K$-corrections (Nugent et al. 2002) are used to transform the rest-frame ( $U B V R I)$ model magnitudes into observer-frame magnitudes. For SALTII, the model is based on a time sequence of rest-frame spectra, and observer-frame magnitudes are computed by convolution with the appropriate filter-response curves.

Since light curve models are defined over a specific wavelength range in the rest frame, one usually checks the rest-frame wavelength $\bar{\lambda}_{\mathrm{f}} /(1+z)$, where $\bar{\lambda}_{\mathrm{f}}$ is the mean wavelength of the observer-frame filter and $z$ is the redshift. If the rest-frame wavelength is outside the valid range of the model, then the corresponding filter is typically ignored. For photo- $z$ applications, this method of ignoring filters clearly cannot be used, since the redshift is not known ahead of time. To be realistic, we should not use this filter-ignoring procedure in the simulations either. Therefore, our simulations use wavelength-extended models that generate fluxes for filters with $\bar{\lambda}_{\mathrm{f}} /(1+z)$ beyond the nominally defined wavelength range. For both MLCS $2 \mathrm{~K} 2$ and SALTII, the rest-frame wavelengths are extended down to $2500 \AA$. For SALTII, the $7000 \AA$ upper limit has been raised to $8700 \AA$. We note that the simulation and fitter use the same wavelength-extended model, and therefore these tests do not probe potential problems if the extended part of the model is wrong.

We have implemented two models of intrinsic SN magnitude variations that produce "anomalous" scatter in the Hubble diagram. The source of anomalous scatter is unknown, and it is not clear if the scatter can be reduced with an improved light curve model, or if this scatter is due to some random physical process such as brightness variations as a function of viewing angle in an asymmetric explosion. For simulations with $10^{4}$ times the nominal exposure time and $z<0.5$, i.e., for which photon noise is negligible, we define the anomalous scatter to be the rms $\left(\mathrm{RMS}_{\mu}\right)$ of the difference between the fitted and generated distance modulus $\left(\mu_{\mathrm{fit}}-\mu_{\mathrm{gen}}\right)$ from the fourparameter light curve fit using spectroscopically determined redshifts. The default model, called "color smearing," introduces an independent magnitude fluctuation in each passband, and the fluctuation is the same for all epochs within each passband. A random number $r_{f}$ from a unit-variance Gaussian distribution is chosen for each passband $f$, and a magnitude fluctuation $\delta m_{f}=r_{f} \sigma_{f}$ is added to the generated magnitude at all epochs. As described in Section 5, a scatter of $\sigma_{f}=0.1 \mathrm{mag}$ is needed in order for the simulated photo- $z$ precision to match that of the data. The resulting Hubble scatter is consistent with that seen in analyses of spectroscopically confirmed data samples; $\mathrm{RMS}_{\mu} \simeq 0.16$ for the SNLS (griz) simulations and $\mathrm{RMS}_{\mu} \simeq$ 0.19 mag for the SDSS-II (gri) simulations. The simulated SNLS scatter is slightly smaller because this survey results in larger $\mathrm{S} / \mathrm{N}$ values. The second model of intrinsic variations is called "coherent luminosity smearing": a coherent random magnitude shift, drawn from a Gaussian with $\sigma_{\text {coh }} \sim 0.15$ mag, is added to the model magnitude for all epochs and passbands. In the coherent smearing method the intrinsic model colors are not varied, and the resulting anomalous scatter is $\mathrm{RMS}_{\mu}=\sigma_{\mathrm{coh}}$.

Although both models of intrinsic magnitude variation result in the expected scatter in the Hubble diagram, only the colorsmearing model can generate the observed photo- $z$ precision in the SDSS-II and SNLS data samples (Section 5). We have not investigated simulations in which both models of intrinsic variation contribute, nor have we investigated variations in the color parameter $\left(R_{V}\right.$ for MLCS $2 \mathrm{~K} 2$ or $\beta$ for SALTII) that could also introduce anomalous scatter.

To simulate non-photometric conditions and varying time intervals between observations due to bad weather, actual observing conditions are used for an existing survey, or an estimate of such conditions for a planned (future) survey. For each simulated observation, the noise is determined from the measured point-spread function (PSF), ${ }^{20}$ zero point, CCD gain, and sky background. Noise from the host-galaxy background is not included. The simulated flux in CCD counts is based on a mag-to-flux zero point and a random fluctuation drawn from the noise estimate. For the SDSS-II and SNLS surveys, a detailed treatment of the search efficiency, including spectroscopic selection effects, is described in Section 6.2 of K09.

The quality of the simulation is illustrated in Figures 1 and 2 for the SDSS-II and SNLS surveys, respectively, using the SN selection requirements described in Section 2. The parameters from each sample have been determined using the conventional fitting method with a fixed spectroscopically determined redshift. Each figure shows data-simulation comparisons for the distributions of spectroscopic redshift, color parameter $\left(A_{V}\right.$ for $\operatorname{MLCS} 2 \mathrm{~K} 2, c$ for SALTII), and shape-luminosity parameter ( $\Delta$ for $\operatorname{MLCS} 2 \mathrm{~K} 2, x_{1}$ for SALTII). The color and shape parameters are defined in Section 4. There is good overall consistency between the measured and simulated distributions.

\footnotetext{
20 The PSF is described by a double-Gaussian function for the SDSS-II and by a single-Gaussian for the other surveys.
} 
No. 1,2010
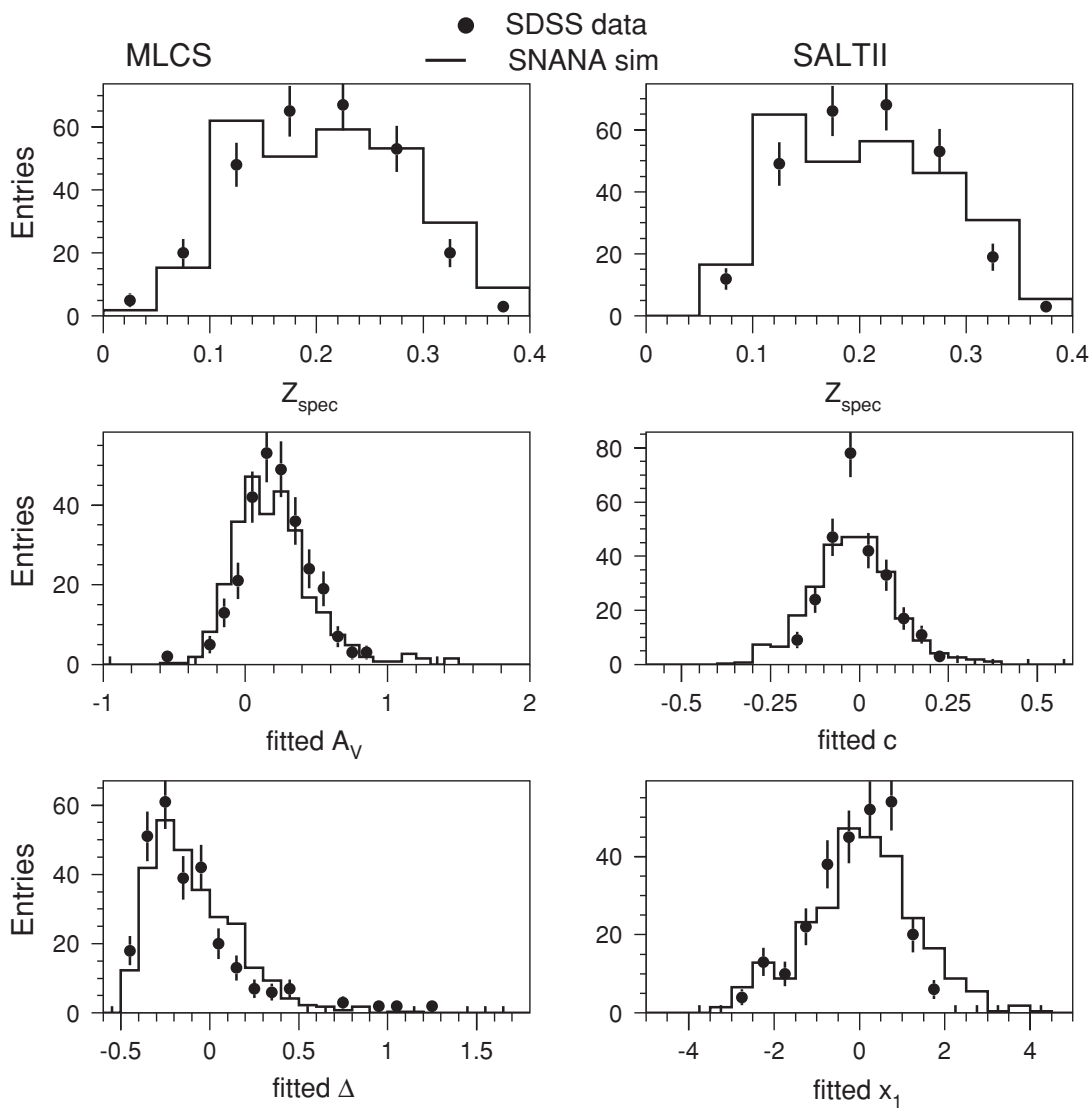

Figure 1. For the SDSS-II SN Survey, comparison of data distributions (dots) with those from the simulation (histograms). Fitted parameters for MLCS2K2 ( $z, A_{V}, \Delta$ ) are on the left and for SALTII $\left(z, c, x_{1}\right)$ on the right. Fits were done with the redshift fixed to the true redshift. The simulated histograms are scaled to have the same number of entries as the data.

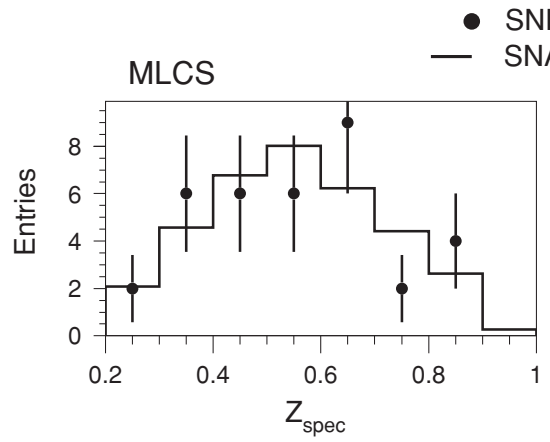

SNLS data

SNANA sim

SALTII
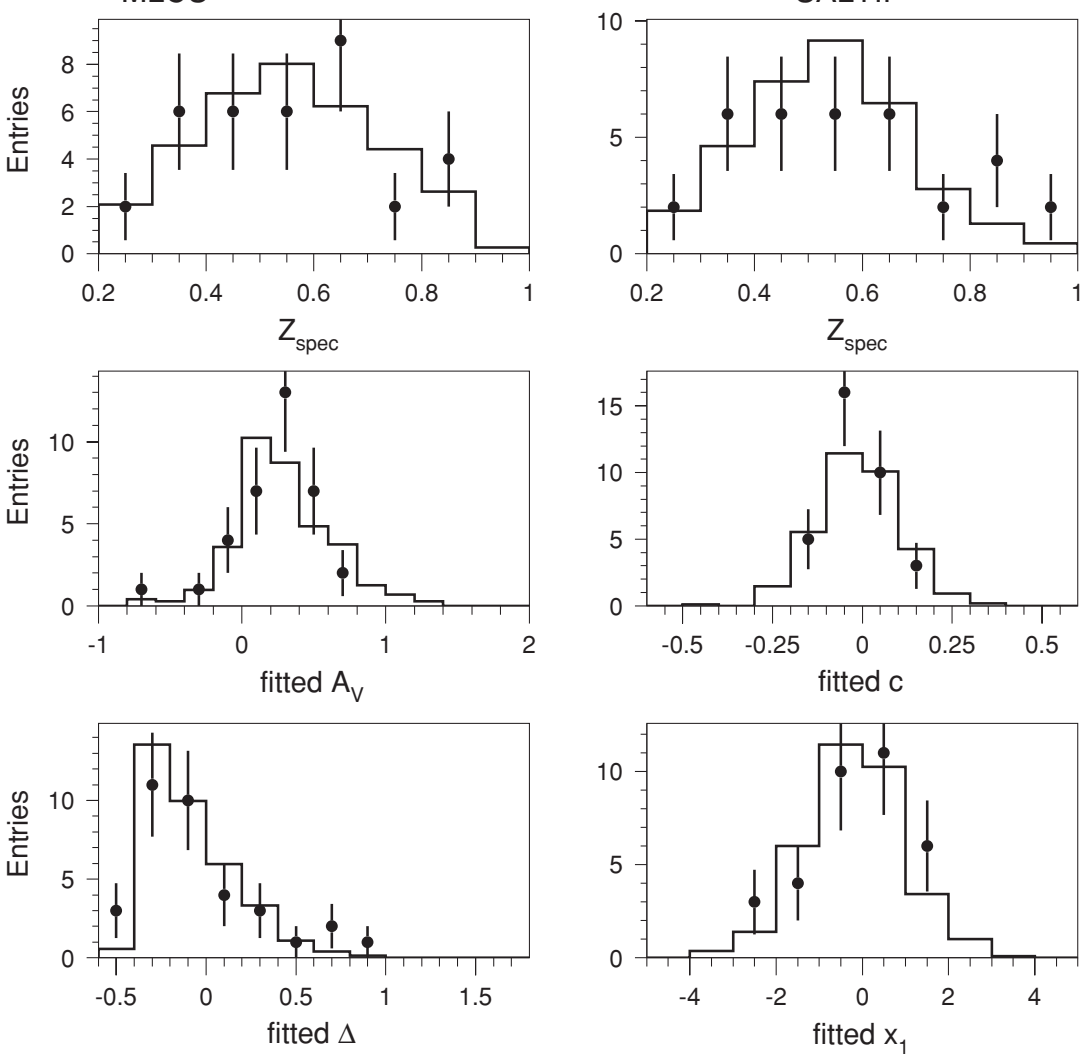

Figure 2. Same as Figure 1 but for the SNLS survey, using the data sample from A06. 


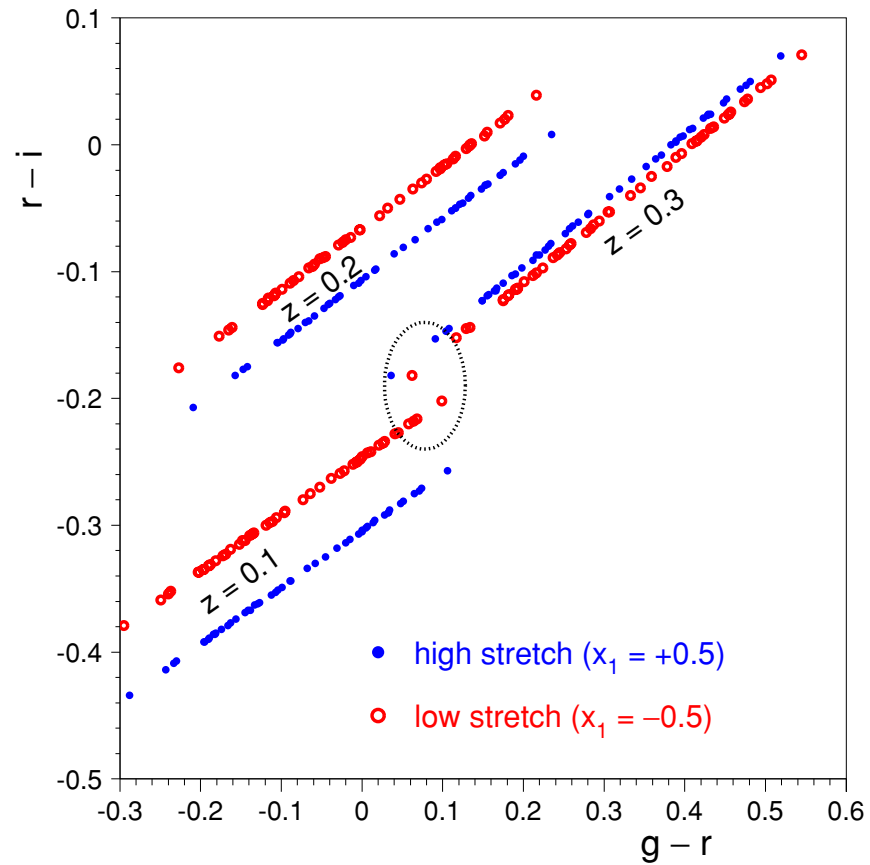

Figure 3. Observer-frame $r-i$ vs. $g-r$ at peak brightness for SNe Ia using the SALTII model. Each set of color bands corresponds to the indicated redshift $(0.1,0.2,0.3)$, and the variation within each band corresponds to variations in the intrinsic SN color (i.e., the SALTII $c$ parameter). The solid and open circles correspond to a high-stretch and low-stretch SN, respectively. The dotted oval shows a degenerate region discussed in the text.

(A color version of this figure is available in the online journal.)

\section{THE LCFIT+Z METHOD}

The general principle behind the SN Ia photo- $z$ determination is illustrated in Figure 3 using colors at the epoch of peak brightness. Increasing the redshift causes uniform reddening at all wavelengths, while intrinsic reddening (or extinction) causes more reddening at bluer wavelengths. A time-dependent light curve model (e.g., MLCS2K2 or SALTII) is used to account for the known color dependence on the light curve shape (commonly known as the stretch) and to use all epochs in order to maximize the photostatics in the photo- $z$ measurement. Since SNe become redder with increasing epoch, any error in the epoch of peak brightness results in the wrong template colors, and hence an increased error in the photo- $z$.

For real observations, the ideal color-color bands in Figure 3 are smeared by photon statistics and by intrinsic SN color variations that are not described by the light curve model. With sufficient smearing, the colors of a very reddened $\mathrm{SN}$ at $z \simeq 0.1$ are degenerate with a blue $\mathrm{SN}$ at $z \simeq 0.3$ (see the region enclosed by dotted oval in Figure 3) and we indeed see this degeneracy in the SDSS-II photo- $z$ measurements. From datasimulation comparisons, we find that smearing from photon statistics does not fully describe the photo- $z$ precision, and we therefore propose that the modeling of intrinsic color variations is not adequate (Section 5).

While host-galaxy photometric redshifts depend on the determination of the $4000 \AA$ break, a similar $\sim 2800 \AA$ break in the SN spectrum has little impact on the photo- $z$ measurement because this feature is either inaccessible at low redshifts, or it is poorly measured at higher redshifts. In current SN Ia models, fluxes at these very blue wavelengths are either ignored, or they are heavily down-weighted relative to the optical bands.
The basic idea of the LCFIT $+Z$ method is to start with a light curve fit model that has four free parameters when the redshift is fixed to an accurately measured value, and simply float the redshift as a fifth fitted parameter. We refer to these methods as MLCS $2 \mathrm{~K} 2+\mathrm{Z}$ and SALTII $+\mathrm{Z}$, where MLCS $2 \mathrm{~K} 2$ and SALTII refer to the conventional models in which the redshift is fixed to a precisely measured value. Compared to the color-based photo- $z$ method, advantages of the LCFIT $+Z$ method include a natural framework for tracking correlations between redshift and distance modulus (Section 5.2), and using all of the light curve information (instead of just peak flux) so that in principle the intrinsic SN color variations can be accounted for. The main advantage of the color-based method is that it works over a broader redshift range, and there is no need to worry about which observer-frame filters map into a valid wavelength range in the rest frame.

For MLCs $2 \mathrm{~K} 2+\mathrm{Z}$, the five fitted parameters (which we denote with the vector $\vec{x}_{5}$ ) are the time of maximum brightness in the (rest frame) $B$ band $\left(t_{0}\right)$, the shape-luminosity parameter $(\Delta)$, the host-galaxy extinction in the $V$ band $\left(A_{V}\right)$, the distance modulus $(\mu)$, and the redshift $\left(z_{\text {phot }}\right)$. We use a flat $A_{V}$ prior and $R_{V}=A_{V} / E(B-V)=2.2$. For SALTII+Z the five parameters are the time of maximum brightness in the rest-frame $B$ band $\left(t_{0}\right)$, the shape-luminosity parameter $\left(x_{1}\right)$, the $B-V$ color $(c)$, the flux normalization $\left(x_{0}\right)$, and the redshift $\left(z_{\text {phot }}\right)$. The following light curve fit $\chi^{2}$ is minimized using MINUIT ${ }^{21}$ :

$$
\chi^{2}=\sum_{i}\left\{\frac{\left[F_{i}^{\text {data }}-F_{i}^{\text {model }}\left(\vec{x}_{5}\right)\right]^{2}}{\sigma_{i}^{2}}+2 \ln \left(\sigma_{i} / \tilde{\sigma}_{i}\right)\right\},
$$

and the corresponding probability $\left(e^{-\chi^{2} / 2}\right)$ is used to marginalize as described in Appendix B. Here, $F_{i}^{\text {data }}$ is the SN flux of the $i$ th observation, $F_{i}^{\text {model }}\left(\vec{x}_{5}\right)$ is the predicted flux using the five model parameters $\left(\vec{x}_{5}\right)$, and $\sigma_{i}^{2}=\sigma_{i \text {,stat }}^{2}+\sigma_{i \text {,model }}^{2}$ is the quadrature sum of the measured and model uncertainties, respectively. The index $i$ runs over all epochs and filters. The second term in Equation (1) accounts for model uncertainties that depend on the rest-frame passband and epoch, which in turn depend on the photo- $z$ value. The model uncertainties are shown in Figure 4 for the rest-frame $U$ and $B$ passbands. The reference uncertainty $\left(\tilde{\sigma}_{i}\right)$ from the first iteration of the fit is used in the denominator so that the second term is close to zero in the second iteration; although the $\tilde{\sigma}_{i}$ do not affect the minimization, these terms reduce the change in the calculated fit probability. As explained below, the $\chi^{2}$ is minimized twice in order to include the appropriate filters and epochs. The minimized values and uncertainties are then used to estimate the integration ranges needed to obtain marginalized results.

In this study, we use the MLCS2K2 and SALTII light curve fitters that have been implemented in the SNANA package. The main reasons for using the SNANA code are (1) exactly the same light curve model is guaranteed to be used in both the light curve fits and in the generated simulations, (2) there are several improvements to the MLCS2K2 light curve fitter as explained in $\mathrm{K} 09$, (3) the photo- $z$ implementation is identical for both models, (4) the SNANA fitter is significantly faster than the original fitting software. To check the SALTII implementation in SNANA, we have repeated the light curve fits and cosmology analysis for the six sample combinations in K09 and find that the dark energy equation of state parameter $w$ is always within a few hundredths

\footnotetext{
21 http://wwwasdoc.web.cern.ch/wwwasdoc/minuit/minmain.html
} 

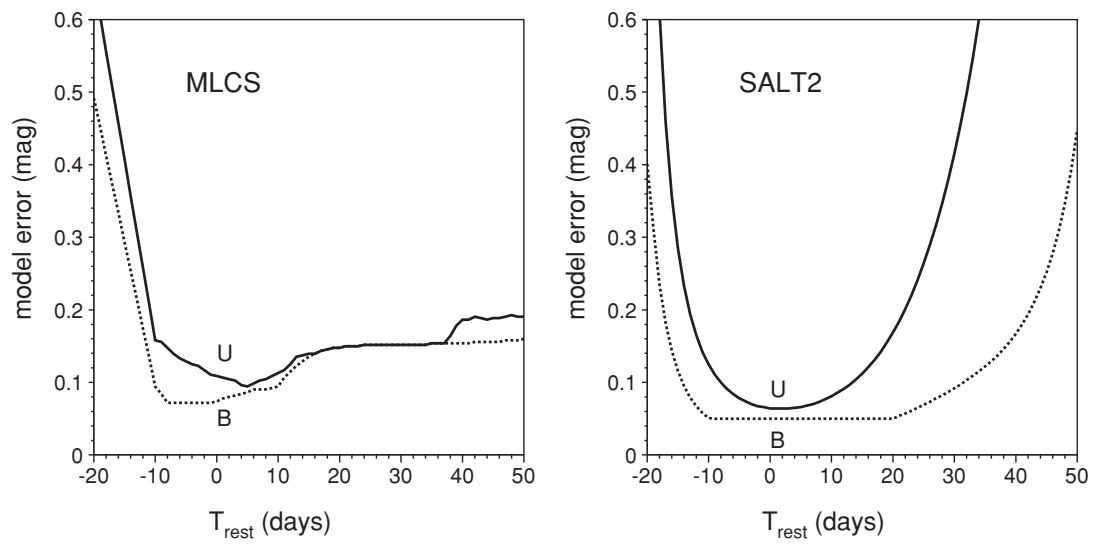

Figure 4. Model uncertainty vs. rest-frame epoch for the $U$ (solid) and $B$ (dashed) passbands.

of the value obtained with the original code; these discrepancies are well below the statistical uncertainties.

Although it is straightforward to include the redshift as a free parameter in the light curve fit, there are subtle pre-fit issues related to the unknown redshift value: (1) SN selection criteria that depend on knowing $T_{\text {rest }}=T_{\mathrm{obs}} /(1+z),{ }^{22}$ such as requiring measurements with a minimum and maximum $T_{\text {rest }}$ value; (2) as noted above, determining which observer-frame filters (with mean wavelength $\bar{\lambda}_{\mathrm{f}}$ ) have $\bar{\lambda}_{\mathrm{f}} /\left(1+z_{\text {phot }}\right)$ within the valid wavelength range of the fitting model; (3) determining the valid rest-frame epoch range for the fitting model; (4) as $\bar{\lambda}_{\mathrm{f}} /\left(1+z_{\text {phot }}\right)$ maps into a different rest-frame filter (for MLCS2K2) there is a discontinuous change in the model error, and therefore the $\chi^{2}$ is not a continuous function of $z_{\text {phot }}$; and (5) determining robust initial fit parameter values. Our treatment of these issues is described in Appendix A.

We end this section with a discussion of the processing time. For the SDSS-II light curves, which have nearly 50 measurements on average, all of the minimization fit iterations take $\sim 1 \mathrm{~s}$ per $\mathrm{SN}$ using MINUIT. The marginalization (Appendix B) takes close to half a minute per $\mathrm{SN}$ using an integration grid of 11 points per fit parameter, or a total of $11^{5}$ integration cells. However, the integration ranges usually need adjustment after marginalizing, and therefore the marginalization typically runs twice, taking nearly a minute per $\mathrm{SN}$. The processing time scales linearly with the number of measurements and as the fifth power of the number of grid points per fit parameter. For the integration grid above, the Monte Carlo Markov Chain technique requires about the same amount of processing time.

Using the SNANA implementation of $\mathrm{MLCS} 2 \mathrm{~K} 2+\mathrm{Z}$ and SALTII+Z, we find that MINUIT gives adequate minimized values, but that the uncertainties are not reliable because of subtle discontinuities in the $\chi^{2}$ derivative with respect to the photo- $z$. We must therefore marginalize in order to get useful uncertainties and covariances. To illustrate the computational issue more clearly, consider an LSST sample of 500,000 SNe Ia. The marginalization for all of the $\mathrm{SNe}$ in this sample requires a total of 1 CPU year. A factor of 100 is probably needed for code development and systematic studies and another factor of several for simulation studies. The total computing needs are therefore a few CPU centuries with today's processors, assuming that $11^{5}$ integration cells give sufficient accuracy. To use the minimization, which reduces the computing needs to a few CPU years,

\footnotetext{
${ }^{22} T_{\text {rest }}$ and $T_{\text {obs }}$ are the rest-frame and observer-frame times in days since
} peak brightness in the $B$ band. the light curve model magnitudes and errors must be continuous functions of redshift, as well as their derivatives.

\section{RESULTS FOR SDSS-II AND SNLS}

Here we present results for the three-season SDSS-II data and the first-season SNLS data described in Section 2, and we compare with results from simulations of those same samples. There are two fit minimizations (Section 4) to determine the appropriate filters to include. The photo- $z$ and distance-modulus results are taken to be the mean of their respective probability distribution functions (pdf's) marginalized over the other fit parameters using a grid of $11^{5}$ integration cells (Appendix B). The uncertainty is taken to be the rms of the pdf. A prior on the host-galaxy photo- $z$ or SN color could potentially improve the precision of the method and reduce the frequency of catastrophic SN photo- $z$ outliers; we have not used such priors here in order to better illustrate the performance of the LCFIT $+\mathrm{Z}$ method on its own.

Following a commonly used practice in the literature, we characterize the precision of the LCFIT $+Z$ photo- $z$ precision with the quantity

$$
\Delta_{z} \equiv\left(z_{\mathrm{phot}}-z_{\mathrm{spec}}\right) /\left(1+z_{\mathrm{spec}}\right),
$$

and we use $\mathrm{RMS}_{\Delta_{2}}$ to denote the root mean square of the distribution of $\Delta_{z}$. The SDSS-II results are shown in Figures 5 and 6 for the MLCS $2 \mathrm{~K} 2+\mathrm{Z}$ and SALTII $\mathrm{Z}$ methods, respectively. There are fewer SNe in the sample fitted with SALTII because the more restrictive rest-frame model wavelength range (2900-7000 ̊̊) rejects $i$-band data for redshifts below about 0.1 ; without $i$ band, these low-redshift SNe fail the requirement of three observerframe filters. The SNLS results are shown in Figures 7 and 8. Distributions from the data and simulated samples are shown side-by-side in these figures, illustrating the reliability of the simulations in predicting the dispersions and bias. Each plot shows the number of SNe satisfying the selection criteria, $\mathrm{RMS}_{\Delta_{\text {, }}}$ (overall and versus $z_{\text {spec }}$ ), and the redshift bias (overall and versus $z_{\text {spec }}$ ). The $\mathrm{RMS}_{\Delta_{z}}$ values are $\sim 0.04$ for both the SDSS-II and SNLS samples.

For the MLCS2K2+Z method applied to the SDSS-II sample (Figure 5), the overall $\Delta_{z}$ bias in the data is notably larger than in the simulation, and there is a redshift-dependent bias that is partially predicted by the simulation. There are two contributions to the $\Delta_{z}$ bias. First, there is a degeneracy between intrinsic reddening (due to extinction or color) and redshift. For redshifts $z_{\text {spec }}>0.3$, the best-fit photo- $z$ is sometimes near 

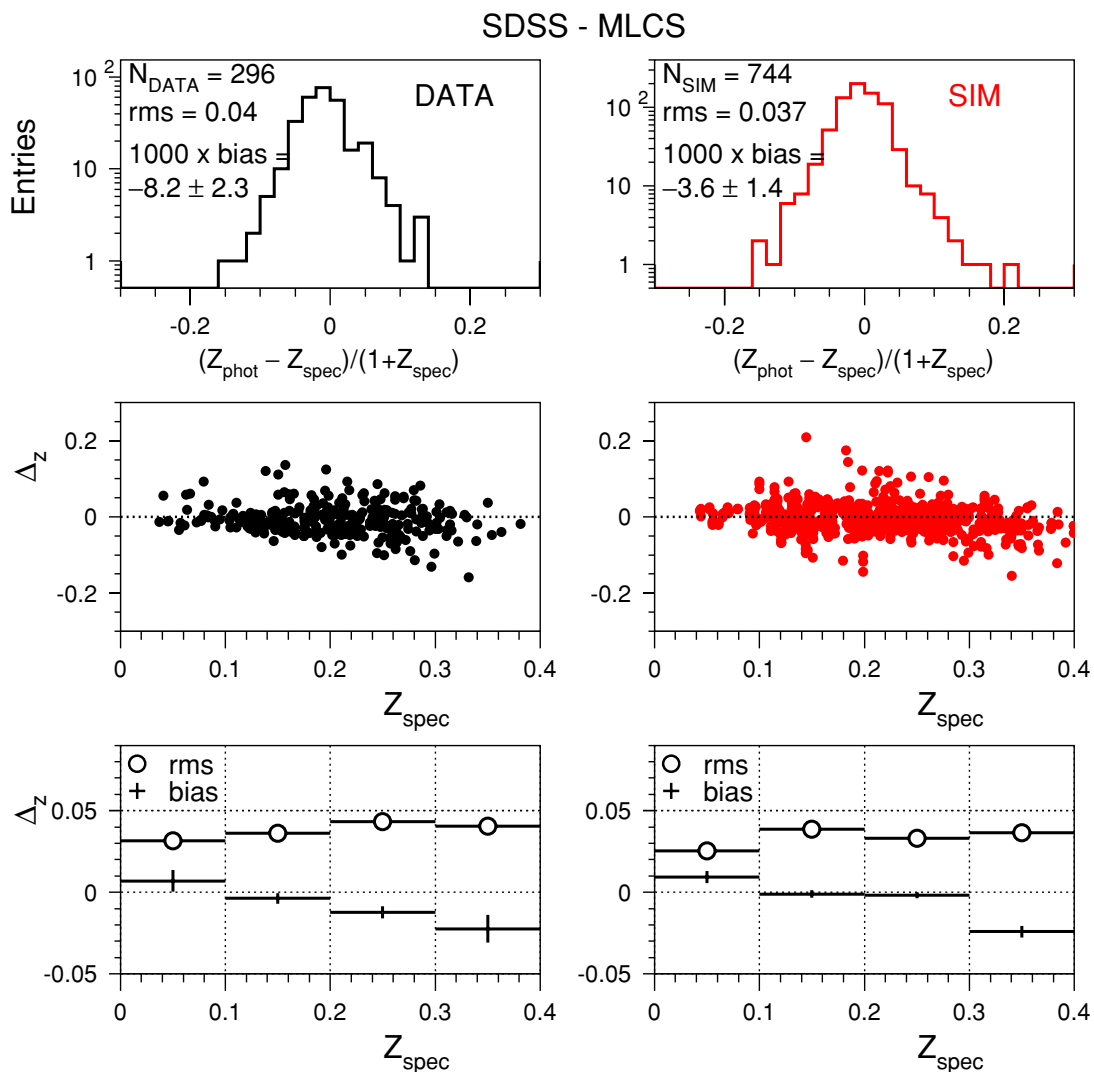

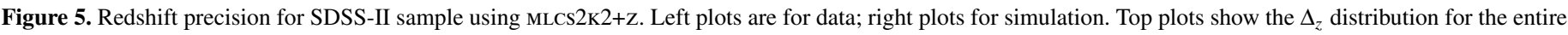
sample; the number of events, rms, and bias are indicated on the plot. Middle plots show $\Delta_{z}$ vs. $z_{\text {spec }}$. Bottom plots show the bias and RMS $\Delta_{z}$ in redshift bins.

(A color version of this figure is available in the online journal.)
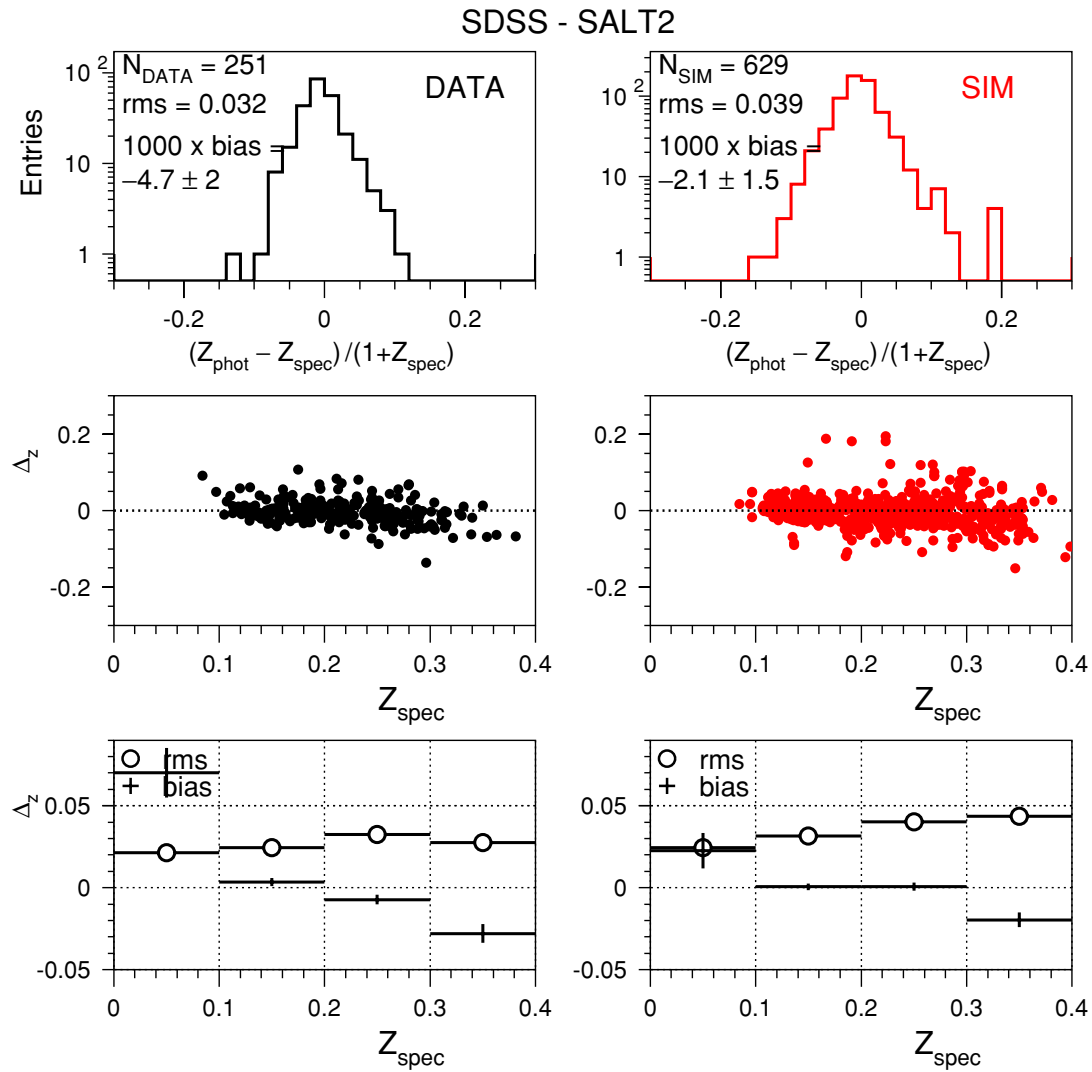

Figure 6. Same as Figure 5, but using SALTII+Z.

(A color version of this figure is available in the online journal.) 
SNLS - MLCS
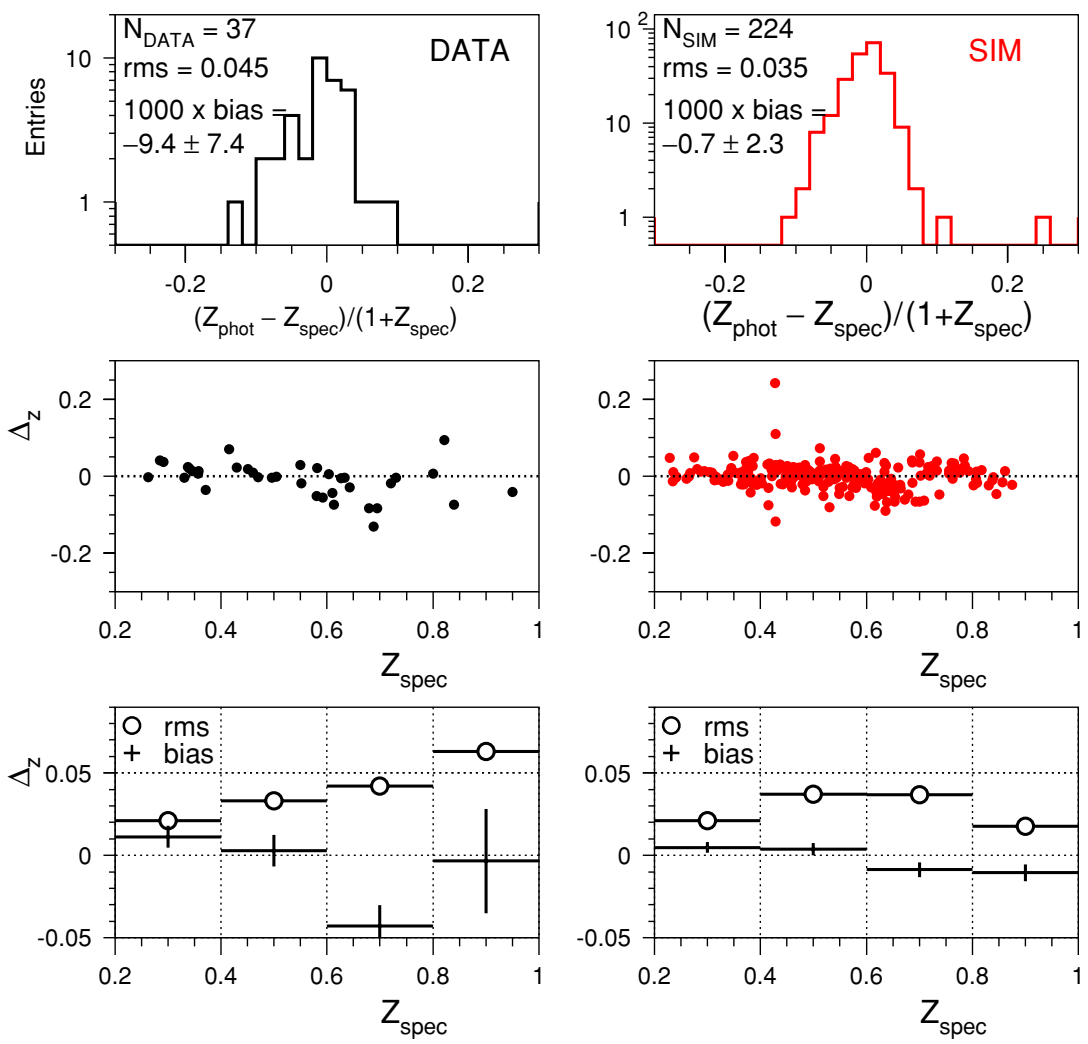

Figure 7. Same as Figure 5, but using the SNLS sample.

(A color version of this figure is available in the online journal.)

SNLS - SALT2
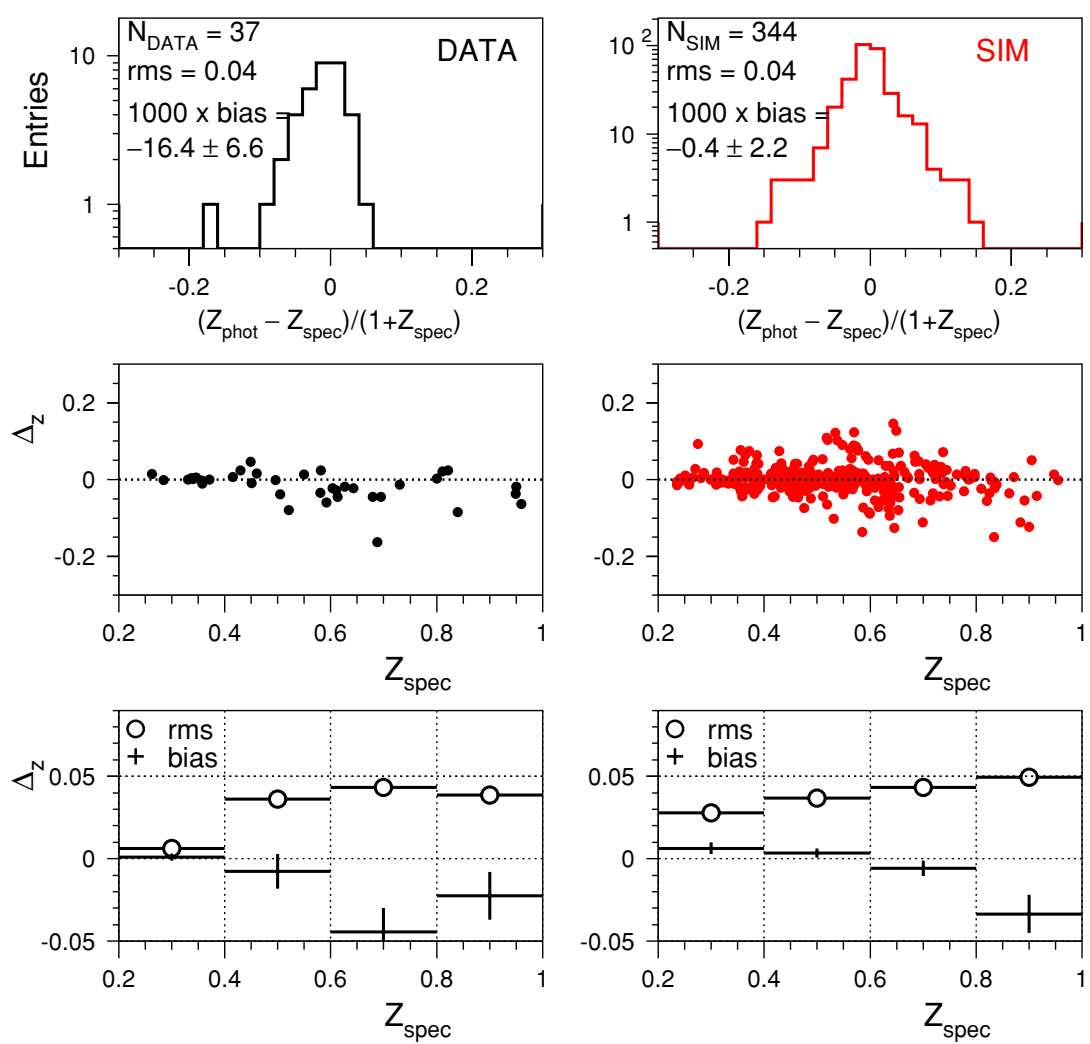

Figure 8. Same as Figure 5, but using the SNLS sample and SALTII+Z.

(A color version of this figure is available in the online journal.) 

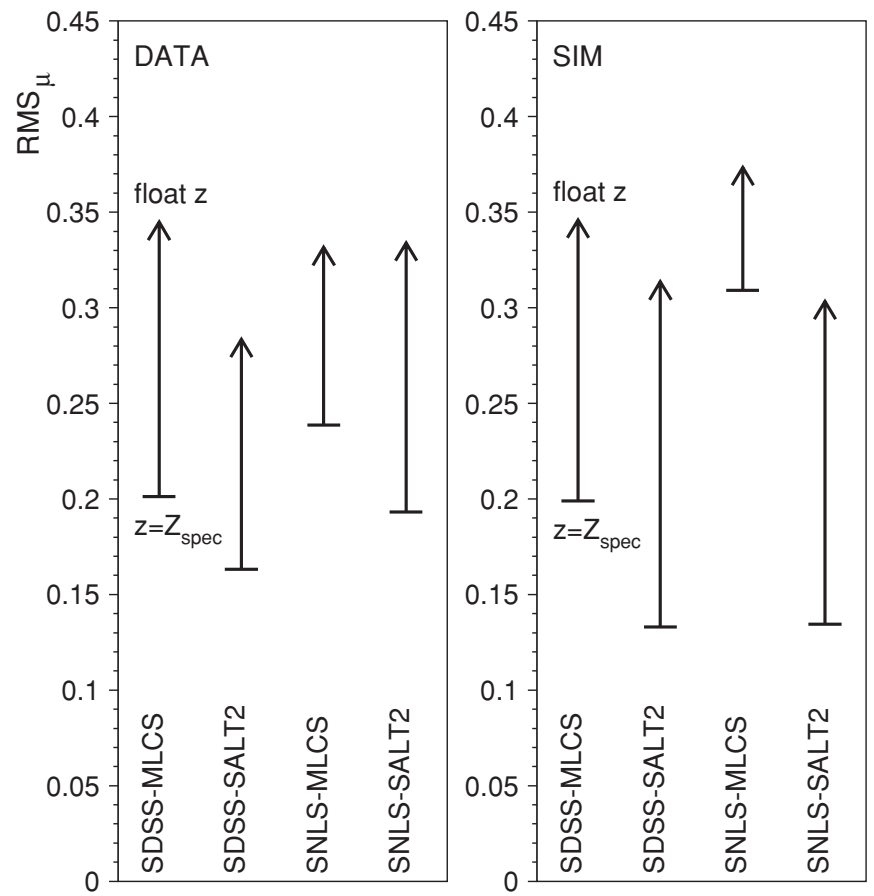

Figure 9. $\mathrm{RMS}_{\mu}$ for four-parameter MLCS $2 \mathrm{~K} 2$ and SALTII fits (bottom of each arrow) and for five-parameter MLCS $2 \mathrm{~K} 2+\mathrm{Z}$ and SALTII $+Z$ fits (top of each arrow) The left panel shows $\mathrm{RMS}_{\mu}$ for the data; the right panel shows the simulations.

$z_{\text {phot }} \sim 0.15$, and the best-fit color corresponds to a very red and intrinsically $\operatorname{dim} \mathrm{SN}$ Ia. This degeneracy is sensitive to the $\mathrm{S} / \mathrm{N}$; the resulting bias is redshift dependent and is well modeled by the simulation.

To potentially identify fits with a catastrophic photo- $z$ error resulting from a strong color-redshift degeneracy, we have looked for a second maximum in the one-dimensional marginalized pdf. For MLCS $2 \mathrm{~K} 2+\mathrm{Z}, \sim 9 \%$ of the fits have a second maximum in both the photo- $z$ and color pdf, and for SALTII+Z the corresponding fraction is $\sim 4 \%$. For both models, the photo- $z$ precision is the same for the subset with a second maximum in the pdf, and therefore this approach cannot be used to identify photo- $z$ outliers in the SDSS-II sample. We also find no correlation between the photo- $z$ uncertainty and catastrophic outliers. Clearly, a reliable host-galaxy photo- $z$ prior will help reduce catastrophic outliers, and this information will be used in future analyses that include spectroscopically unconfirmed SNe Ia in the Hubble diagram.

The second contribution to the $\Delta_{z}$ bias is related to the $U$-band anomaly discussed in $\mathrm{K} 09$, and this source of bias is not modeled in the simulation. The sub-sample with $z>0.2$, where the observer-frame $g$ band corresponds to the rest-frame $U V$ region, has a bias larger than the average. The sub-sample with $z<0.2$ has a much smaller bias.

For cosmological applications, it is of interest to study how the use of photo- $z$ 's in place of spectroscopic redshifts impacts the determination of SN distances. Distance modulus $(\mu)$ dispersions for fits with both spectroscopic and photometric redshifts are shown in Figure 9, where $\mathrm{RMS}_{\mu}$ is the rms scatter of the distribution of $\mu_{\text {fit }}-\mu_{\text {ref }}$. The reference distance modulus $\left(\mu_{\text {ref }}\right)$ is calculated from the spectroscopic redshift and the same standard cosmology used in the simulation: $w=-1, \Omega_{\mathrm{M}}=0.3$, $\Omega_{\Lambda}=0.7$. For MLCS $2 \mathrm{~K} 2, \mu_{\mathrm{fit}}$ is the fitted distance modulus. For SALTII, $\mu_{\text {fit }}$ is defined to be

$$
\mu_{\mathrm{fit}}^{\mathrm{SALT} 2} \simeq 30-2.5 \log _{10}\left(x_{0}\right)+\alpha \cdot x_{1}-\beta \cdot c,
$$

Table 1

Photo- $z$ Precision $\sigma_{\Delta_{z}}{ }^{\mathrm{a}}$ for Data and Simulations

\begin{tabular}{lcccc}
\hline \hline Sample & $\begin{array}{c}\text { SDSS-II } \\
(\text { MLCS2K2+Z) }\end{array}$ & $\begin{array}{c}\text { SDSS-II } \\
(\text { SALTII+Z) }\end{array}$ & $\begin{array}{c}\text { SNLS } \\
(\text { MLCS2K2+Z) }\end{array}$ & $\begin{array}{c}\text { SNLS } \\
(\text { SALTII+Z) }\end{array}$ \\
\hline DATA & $0.031 \pm 0.003$ & $0.027 \pm 0.002$ & $0.051 \pm 0.020$ & $0.032 \pm 0.006$ \\
SIM $^{\mathrm{b}}$ & $0.030 \pm 0.001$ & $0.030 \pm 0.001$ & $0.027 \pm 0.002$ & $0.028 \pm 0.002$ \\
SIM $^{\mathrm{c}}$ & $0.020 \pm 0.001$ & $0.017 \pm 0.001$ & $0.021 \pm 0.001$ & $0.014 \pm 0.001$
\end{tabular}

Notes.

${ }^{\mathrm{a}} \Delta_{z} \equiv\left(z_{\text {phot }}-z_{\text {spec }}\right) /\left(1+z_{\text {spec }}\right)$

${ }^{\mathrm{b}}$ Uses color-smearing model with $\sigma_{f}=0.1 \mathrm{mag}$.

${ }^{\mathrm{c}}$ Uses 0.2 mag coherent mag-smearing.

where $\alpha=0.11$ and $\beta=2.6$ are fixed parameters from the simulation. Note that the particular choice of $\alpha$ and $\beta$ does not affect the $\mu$ dispersion. Compared to the four-parameter light curve fits using spectroscopic redshifts, the LCFIT $+Z$ method increases $\mathrm{RMS}_{\mu}$ by $0.1-0.2 \mathrm{mag}$.

To gauge the appropriate level of intrinsic magnitude variations in the simulation, we compare the $\Delta_{z}$ precision in the data to that in two different simulated samples. The first sample is generated with $0.1 \mathrm{mag}$ color smearing, and the second sample is generated with 0.2 mag coherent smearing. The width of a Gaussian fit to the $\Delta_{z}$ distribution $\left(\sigma_{\Delta_{z}}\right)$ is used instead of $\mathrm{RMS}_{\Delta_{z}}$ to reduce sensitivity to outliers. The $\sigma_{\Delta_{z}}$ results are shown in Table 1 for the SDSS-II and SNLS data and for the simulated samples. Using coherent smearing, the simulated photo- $z$ precision is significantly better than that of the data, while the colorsmearing model matches the data well. For the small SNLS data sample fitted with MLCS $2 \mathrm{~K} 2+\mathrm{Z}$, the large uncertainty on $\sigma_{\Delta_{z}}$ is due to a statistical anomaly in the distribution that results in a poor fit to a Gaussian.

This empirical estimate of random intrinsic color dispersion needed in the simulation does not necessarily suggest that there are random color variations in SN light curves, but rather that there are additional sources of color variation that are not captured by the light curve models. Using the nearby SN Ia sample $(z<0.1)$, Nobili \& Goobar (2008) also found evidence for intrinsic color dispersion. They fit the SNe with a light curve model that includes many more color parameters than MLCS $2 \mathrm{~K} 2$ or SALTII, and their estimate of the color dispersion is considerably smaller than our empirical estimate based on matching the photo- $z$ precision.

\subsection{Comparison with Recent SNLS Photo-z Results}

Using data and simulations for the SNLS, we compare our photo- $z$ precision with recent results from PD09. They use the SALTII $+Z$ method in a manner very similar to ours. The differences between our method and theirs are (1) they use all four griz filters, while we use only those filters that correspond to the valid rest-frame wavelength range; (2) their initial parameter scan is in redshift only $(\Delta z=0.1$ bins), while our initial scan is over a two-dimensional grid of redshift $(\Delta z=0.04$ bins) and color $(\Delta c=0.2$ bins $)$; (3) they impose priors on the color and redshift, while we do not use priors; and (4) they impose less restrictive light curve selection requirements, including the addition of photometrically identified SNe Ia (i.e., spectroscopically unconfirmed $\mathrm{SNe}$ ). The trade-off between the use of priors versus selection criteria mainly affects the rate of catastrophic photo- $z$ outliers. Our choice of using flat priors is intended to better illustrate the performance of the SALTII+Z method on higher-quality light curves. While the relaxed cuts in PD09 have the advantage of increasing the sample size, 
MLCS2k2+Z Fit Correlations for SDSS
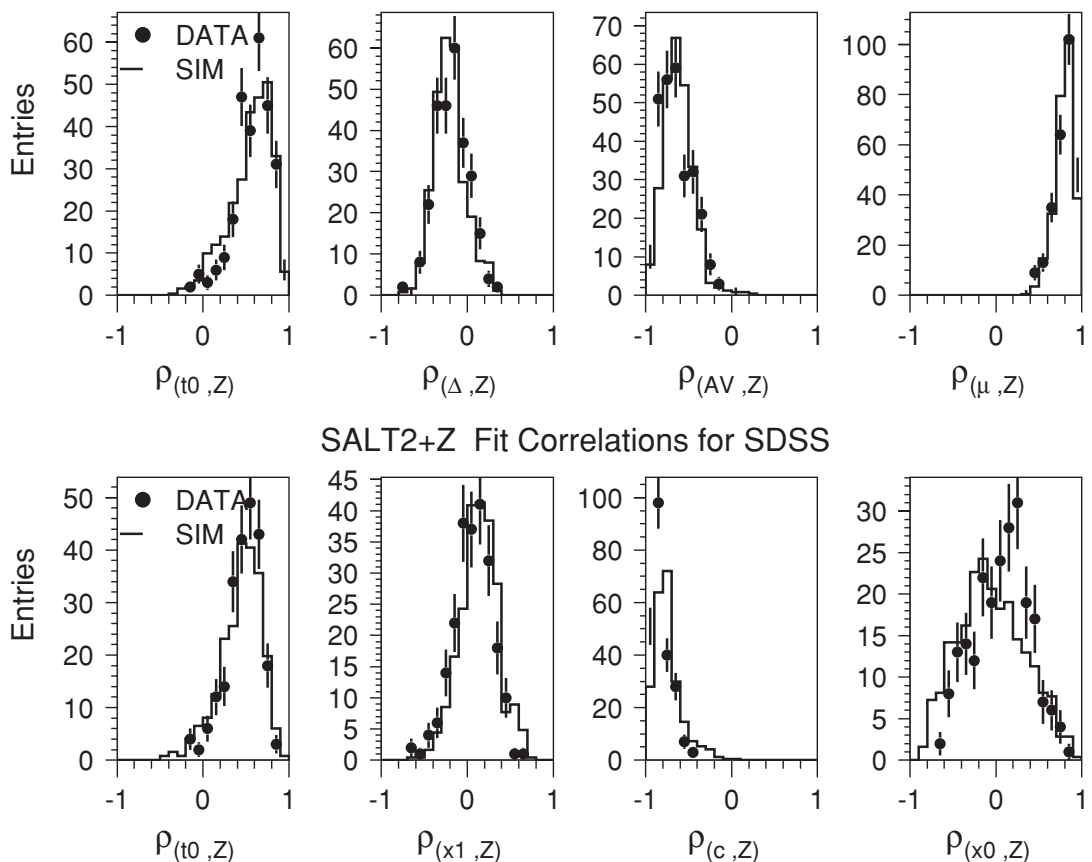

SALT2 $+Z$ Fit Correlations for SDSS
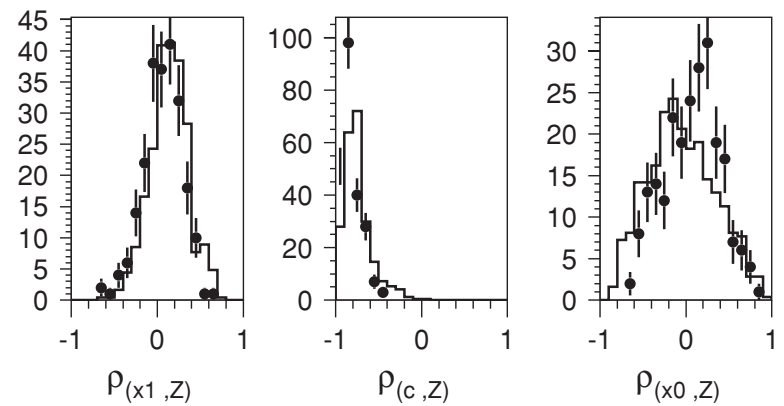

Figure 10. Reduced correlations $(\rho)$ between the photo- $z(Z)$ and the other four light curve fit parameters as indicated in the title of each plot. Top plots are for the SDSS-II sample using MLCS2K2+Z; bottom plots are for the SALTII+Z method. The data are shown by dots; the simulation is shown by the histogram.

using a prior on color (or on host-galaxy extinction) requires a detailed understanding of the underlying color distribution and survey selection function. The optimal choice between priors and selection criteria is not addressed here and will need further study.

The PD09 sample is based on nearly 300 SNe Ia corresponding to the first three seasons of SNLS, while we use the publicly available sample from A06. To make the selection criteria more similar for this comparison, we have relaxed our requirement on the maximum $\mathrm{S} / \mathrm{N}$ : three filters must have at least one measurement each with $\mathrm{S} / \mathrm{N}>5$ (instead of 8). Our modified selection results in $55 \mathrm{SNe}$ Ia: 13 with $z<0.45$ and 42 with $z>0.45$.

To evaluate the photo- $z$ precision, we use the PD09 metric $\sigma_{\Delta z /(1+z)} \equiv 1.48 \times$ median $\left|\Delta_{z}\right|$, where $\Delta_{z}$ is defined in Equation (2). This quantity is much closer to the Gaussian sigma of $\Delta_{z}$ than to $\mathrm{RMS}_{\Delta_{z}}$. To quantify the rate of catastrophic photo- $z$ outliers, we define $\eta_{x}$ as the fraction of SNe with a photo- $z$ that satisfies $\left|\Delta_{z}\right|>x$, and we follow PD09 in using $x=0.15$.

Table 2 compares our precision metrics with those in PD09. We also give the $\sigma_{\Delta z /(1+z)}$ breakdown for the low-redshift $\left(z_{\text {spec }}<\right.$ $0.45)$ and high-redshift $\left(z_{\text {spec }}>0.45\right)$ ranges. We caution that these comparisons are based on different data samples, different selection criteria, and different priors. For the data comparison, the two analyses are reasonably consistent in both $\sigma_{\Delta z /(1+z)}$ and $\eta_{0.15}$ for both redshift ranges. For the simulation comparison there is a subtle disagreement. The PD09 simulation, which uses coherent mag-smearing, underestimates the scatter in the lowredshift range but accurately predicts the precision in the highredshift range. Our simulation using coherent mag-smearing underestimates $\sigma_{\Delta z /(1+z)}$ (in PD09) for both redshift ranges, but our simulation based on color smearing predicts $\sigma_{\Delta z /(1+z)}$ fairly well, perhaps with a slight overestimate of the scatter. Our simulation supports our earlier conclusion that color smearing is needed to model the photo- $z$ precision; the PD09 simulation supports our conclusion in the low-redshift range but not in the high-redshift range. Finally, our simulation underestimates the
Table 2

Photo- $z$ Precision for SNLS Data and Simulation

\begin{tabular}{lllc}
\hline \hline \multicolumn{1}{c}{ Reference } & $\begin{array}{c}\sigma_{\Delta z /(1+z)} \\
(z<0.45)\end{array}$ & $\begin{array}{c}\sigma_{\Delta z /(1+z)} \\
(z>0.45)\end{array}$ & $\begin{array}{c}\eta_{0.15^{\mathrm{a}}} \\
(\mathrm{all} z)\end{array}$ \\
\hline PD09 using & & & \\
DATA $^{\mathrm{b}}$ (three seasons) & 0.016 & 0.025 & 0.014 \\
SIM $^{\mathrm{b}}$ & 0.006 & 0.027 & 0.010 \\
\hline This work using & & & \\
A06 DATA & $0.005(5)$ & $0.036(7)$ & $0.02(2)$ \\
SIM (coherent smear) & $0.004(2)$ & $0.016(1)$ & $0.002(2)$ \\
SIM (color smear) & $0.019(3)$ & $0.030(3)$ & $0(2)$ \\
\hline
\end{tabular}

Notes. Results for PD09 and this work are shown. Our results include the uncertainty in parentheses.

${ }^{\mathrm{a}} \eta_{0.15}=$ fraction of SNe with $\left|z_{\text {phot }}-z_{\text {spec }}\right| /\left(1+z_{\text {spec }}\right)>0.15$.

${ }^{\mathrm{b}}$ See $z_{\text {pho }}{ }^{\mathrm{b}}$ columns in Table 1 of PD09.

fraction of catastrophic outliers $\left(\eta_{0.15} \sim 0.002\right)$, while the PD09 simulation gives good agreement with the data $\left(\eta_{0.15} \sim 0.01\right)$.

\subsection{Photo-z Correlations}

Here we briefly discuss photo- $z$ correlations that should be propagated in a Hubble diagram analysis. Using the LCFIT $+Z$ results from the SDSS-II sample, Figure 10 shows reduced correlations $(\rho)$ between the photo- $z$ and each of the other four light curve fit parameters. The MLCs $2 \mathrm{~K} 2+\mathrm{Z}$ photo- $z$ correlation with $t_{0}$ and distance modulus (upper plots) are both peaked at large positive values, the correlation with extinction $\left(A_{V}\right)$ is negative, and there is little correlation with the shape parameter $\Delta$. For SALTII+Z (lower plots) the photo- $z$ correlations with $t_{0}$, shape/stretch parameter $\left(x_{1}\right)$, and color are qualitatively similar to those based on the MLCs $2 \mathrm{~K} 2+\mathrm{Z}$ method. The $\rho_{(x 0, z)}$ correlation has a very broad distribution with an average near zero. For MLCS $2 \mathrm{~K} 2+\mathrm{Z}$, the simulated distributions match the data well, while for SALTII+Z there is a slight discrepancy in the 
distributions of $\rho_{(c, z)}$ and $\rho_{x 0, z}$. This discrepancy occurs only for $z_{\text {spec }}>0.2$ and could be an artifact of the subset with smaller $\mathrm{S} / \mathrm{N}$.

\section{PREDICTIONS FOR LSST}

Since we have demonstrated that the SNANA simulation can be used to reliably determine the photo- $z$ precision for the SDSSII and SNLS samples, we now turn our attention to forecasts for the LSST survey. LSST will discover far more SNe than spectroscopic resources can target, and photometric methods will be needed to determine both the redshift and SN type for the majority of events. Here we determine the precision (bias and rms) on the photo- $z$ and distance modulus using the LCFIT $+Z$ method on more than $10^{4}$ simulated SNe Ia (after selection requirements) corresponding to the LSST-DEEP and LSST-MAIN surveys. Contamination from non-Ia SNe and the resulting precision in cosmological parameters will be presented in a future work.

The DEEP survey comprises seven fields, each covering nearly $10 \mathrm{deg}^{2}$, that is densely time-sampled in all of the ugrizY LSST filters. After rejecting passbands with invalid $\bar{\lambda}_{\mathrm{f}} /\left(1+z_{\text {phot }}\right)$, the average number of observations per SN Ia used in the light curve fit is 66. The MAIN survey covers more than 20,000 $\mathrm{deg}^{2}$ but is not optimized for SN observations, so the light curve sampling often has large temporal gaps for each filter. The mean number of fitted observations per SN for the MAIN survey is 20 , more than a factor of 3 fewer compared to the DEEP fields. More details about the LSST are given in Ivezić et al. (2008) and LSST Science Collaborations (2009). Due to the extensive computing resources needed to marginalize these large LSST samples, we have only performed the minimizations that are adequate for determining central values for the fitted parameters.

To simulate observing conditions, we use the output of version OPSIM1.29 of the LSST Operations Cadence Simulator (Delgado et al. 2006 and Section 3.1 of LSST Science Collaborations 2009) for the cadence, sky noise, and 5o limiting magnitude for each measurement. For each observation, the SNANA simulation requires a zero point $\left(Z_{\text {p.e. }}\right)$ to translate the simulated SN magnitude $(m)$ into an observed CCD flux measured in photoelectrons, $F=10^{-0.4\left(m-Z_{\text {p.e. }}\right)}$. In terms of the OPSIM1.29 parameters, we calculate this zero point to be

$$
\begin{aligned}
Z_{\text {p.e. }}= & 2 \mathcal{M}_{5 \sigma p s}-\mathcal{M}_{\text {sky }}+2.5 \log _{10}\left(A \cdot(\mathrm{S} / \mathrm{N})^{2}\right) \\
& +2.5 \log _{10}\left[1+A^{-1} \times 10^{0.4\left(\mathcal{M}_{\text {sky }}-\mathcal{M}_{5 \sigma p s}\right)}\right],
\end{aligned}
$$

where $\mathcal{M}_{5 \sigma p s}$ is the $5 \sigma$ limiting magnitude, $\mathcal{M}_{\text {sky }}$ is the Perry sky brightness (mag $\left.\operatorname{arcsec}^{-2}\right), A=\left[2 \pi \int[\operatorname{PSF}(r)]^{2} r d r\right]^{-1}=$ $(1.51 \cdot \mathrm{FWHM})^{2}$ is the effective aperture (in $\operatorname{arcsec}^{2}$ ) where FWHM describes the seeing, and $\mathrm{S} / \mathrm{N}=5$ is the signal-tonoise ratio corresponding to $\mathcal{M}_{5 \sigma p s}$.

For this study, we carry out the light curve fits both with and without a host-galaxy photo-z prior; no other priors are used. The host-galaxy prior is determined from the Bayesian Photometric Redshift Estimation (BPZ) technique (Benitez 2000) applied to a preliminary set of simulated galaxies. The galaxy colors and luminosities are generated to match observed distributions as a function of redshift. For more details, see Section 3.8 of LSST Science Collaborations (2009). The signal to noise as a function of apparent magnitude for the host-galaxy simulation is based on co-added exposures for 10 years of the MAIN survey, and photo-z's are determined for galaxies with $r$ magnitudes down to 25 . The average host-galaxy photo- $z$ precision from BPZ is

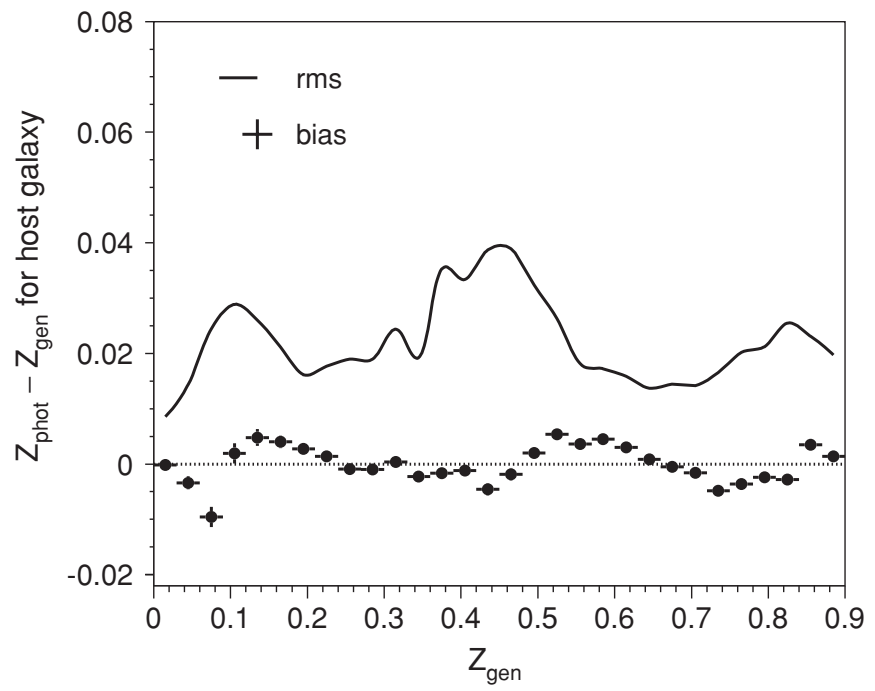

Figure 11. For $6 \times 10^{4}$ simulated LSST host galaxies, BPZ bias (crosses) and rms (curve) of $z_{\text {phot }}-z_{\text {gen }}$ vs. the true host-galaxy redshift $\left(z_{\text {gen }}\right)$ in redshift bins of width $\Delta z=0.03$.

0.02 , and there are some variations with redshift as shown in Figure 11. The $z_{\text {phot }}-z_{\text {gen }}$ bias versus redshift has wiggles of order 0.005 , but the true bias could be larger if the SN host galaxies are not a random subset of the galaxies used for photo$z$ training. Note that in this LSST discussion of the host galaxy and $\mathrm{SNe}$ Ia, we characterize the photo- $z$ precision in terms of $z_{\text {phot }}-z_{\text {gen }}$ instead of $\Delta_{z}$.

These host-galaxy photo- $z$ values are stored in a library for the SNANA simulation. For each simulated SN with true redshift $z_{\mathrm{SN}}$, the host galaxy with true redshift $\left(z_{\mathrm{gal}}\right)$ closest to $z_{\mathrm{SN}}$ is selected. The corresponding host-galaxy photo- $z$ is then scaled by the ratio $z_{\mathrm{SN}} / z_{\text {gal }}$ to correct for the slight redshift mismatch between the SN and the host galaxy. The scaled host-galaxy photo- $z$ and its uncertainty are used to impose a Gaussian prior in $\mathrm{LCFIT}+Z$.

To ensure well-sampled light curves for the LCFIT $+Z$ method, we apply the following selection requirements to the simulated LSST SN data: (1) at least two filters with a measurement satisfying $T_{\text {rest }}<-5$ days; (2) at least two filters with a measurement satisfying $T_{\text {rest }}>+20$ days; (3) largest rest-frame gap (that overlaps -5 to +20 days) is $<15$ days; (4) at least three observer-frame filters have an epoch with $\mathrm{S} / \mathrm{N}>10$; and (5) the light curve fit probability satisfies $P_{\chi^{2}}>0.02$. The requirement of at least two filters (cuts (2) and (3)) removes poorly sampled light curves predominantly from the MAIN survey. Using these requirements, the number of SNe per year is 1900 and $5 \times 10^{4}$ for the DEEP and MAIN surveys, respectively. We note that these selection requirements are based on educated guesses rather than an optimization procedure. Example light curves from the DEEP and MAIN surveys are shown in Figure 12.

As a test of the fitting software, we first simulate ideal DEEPfield samples with no intrinsic mag-smearing and the exposure time artificially increased by a factor of $10^{4}$ compared to the nominal exposure. The resulting photo- $z$ bias is less than 0.001 at all redshifts for both MLCS $2 \mathrm{~K} 2+\mathrm{Z}$ and SALTII+Z, and the photo$z$ dispersion (rms) is less than 0.003 . The bias on the distance modulus varies between 0 and 0.01 mag for $\operatorname{MLCS} 2 \mathrm{~K} 2+Z$, and is less than $\pm 0.005 \mathrm{mag}$ for sALTII+Z. The distance modulus dispersion is $\sim 0.02 \mathrm{mag}$ for both fitting models. 

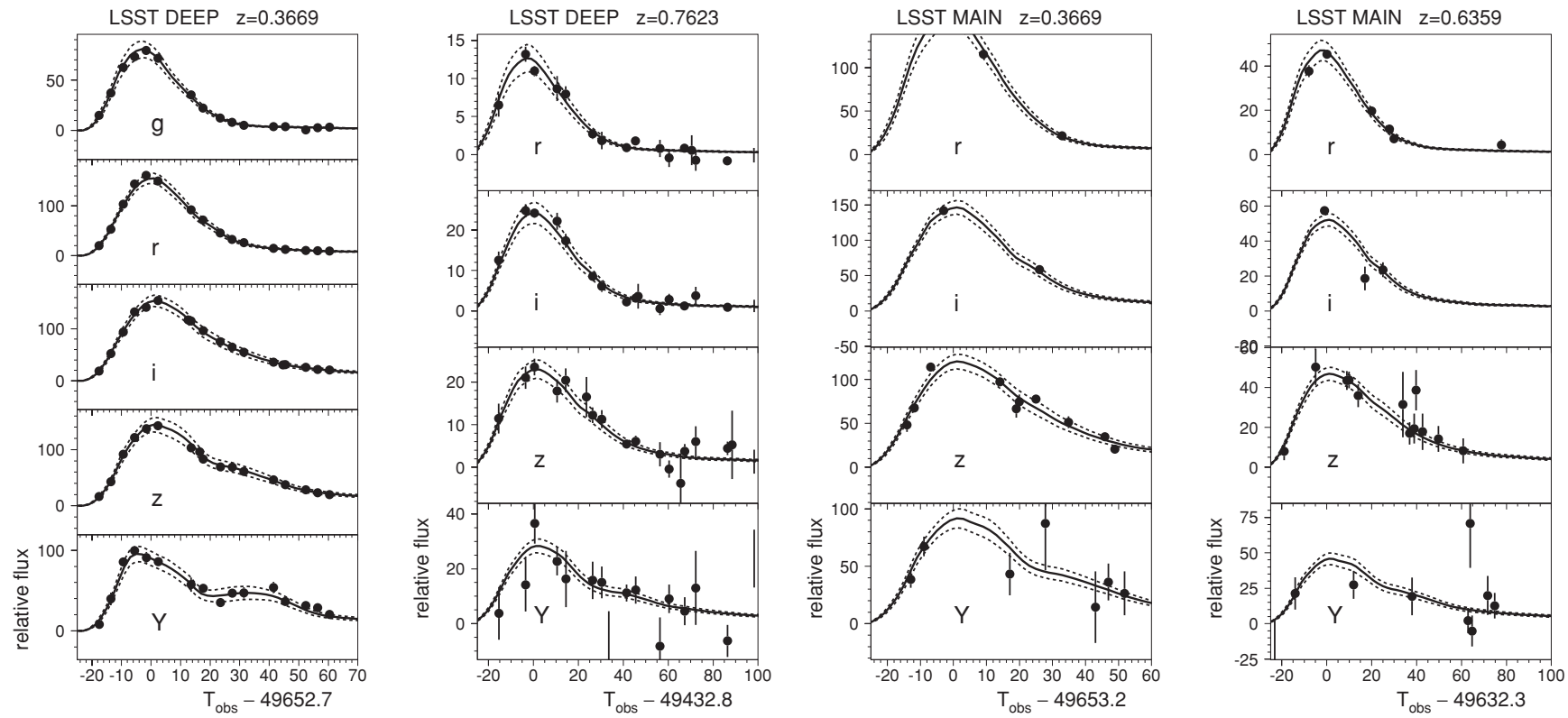

Figure 12. Left two panels show typical SN Ia light curves simulated for the LSST-DEEP fields; right two panels show typical light curves for the LSST-MAIN fields. The redshift is indicated on the top of each plot. Dots are simulated fluxes, the solid curve is the best-fit MLCs $2 \mathrm{~K} 2+\mathrm{Z}$ model, and the dashed curves are the $\pm 1 \sigma$ error bands for the model.
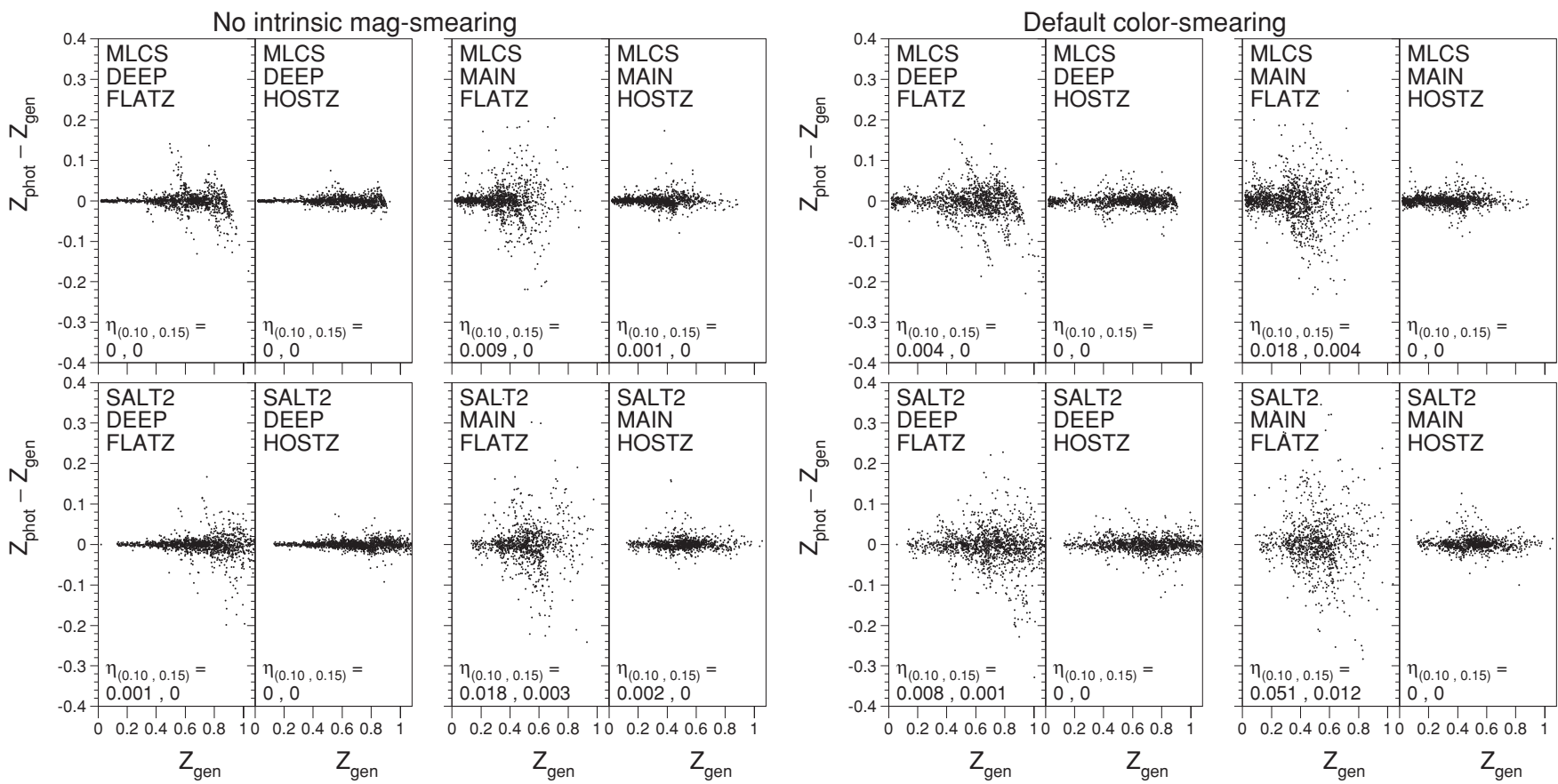

Figure 13. $z_{\text {phot }}-z_{\text {gen }}$ residuals vs. $z_{\text {gen }}$ for the model (мLCS2к2or SALTII), survey (DEEP or MAIN), and photo- $z$ prior (host galaxy or flat) indicated in each panel for LSST simulations. Each pair of plots compares residuals with no photo-z prior (FLATZ) to residuals with host-galaxy photo- $z$ prior (HOSTZ). The fraction of catastrophic outliers $\left(\eta_{0.10,0.15}\right)$, indicated in each panel, is defined in the text. The simulations are generated without intrinsic mag-smearing (left plots) and with the default color-smearing model (right plots).

\subsection{Results for LSST Simulations}

The SN photo- $z$ residuals as a function of redshift are shown in Figure 13 for a simulation without intrinsic magnitude fluctuations and for a simulation using the same intrinsic color fluctuations needed to match the photo-z precision for the SDSSII and SNLS data samples (Section 5). Each panel shows the residuals as a function of fitting method (MLCS $2 \mathrm{~K} 2+\mathrm{Z}$ or SALTII+Z), survey field (DEEP or MAIN) and redshift prior (flat or host-galaxy photo-z). With no intrinsic fluctuations, the most notable effects are (1) with a flat redshift prior, the extreme photo- $z$ outliers extend up to $\left|z_{\text {phot }}-z_{\text {gen }}\right| \sim 0.2$ and (2) the hostgalaxy photo-z prior significantly reduces the number of outliers. With default color fluctuations, there is a notable increase in the photo- $z$ outliers. In both cases, the redshift range is higher for SALTII $+Z$, because it extends to a lower rest-frame wavelength $(2900 \AA)$ than MLCS2K2 (3200 ̊).

We quantify the rate of catastrophic outliers using $\eta_{0.15}$ and $\eta_{0.10}$ (see Section 5.1). With $\sim 10^{4} \mathrm{SNe}$ per sample, the approximate uncertainty is $\sigma_{\eta} \simeq \sqrt{\eta} / 100$. Without intrinsic 
MLCS Coherent mag-smearing in sim

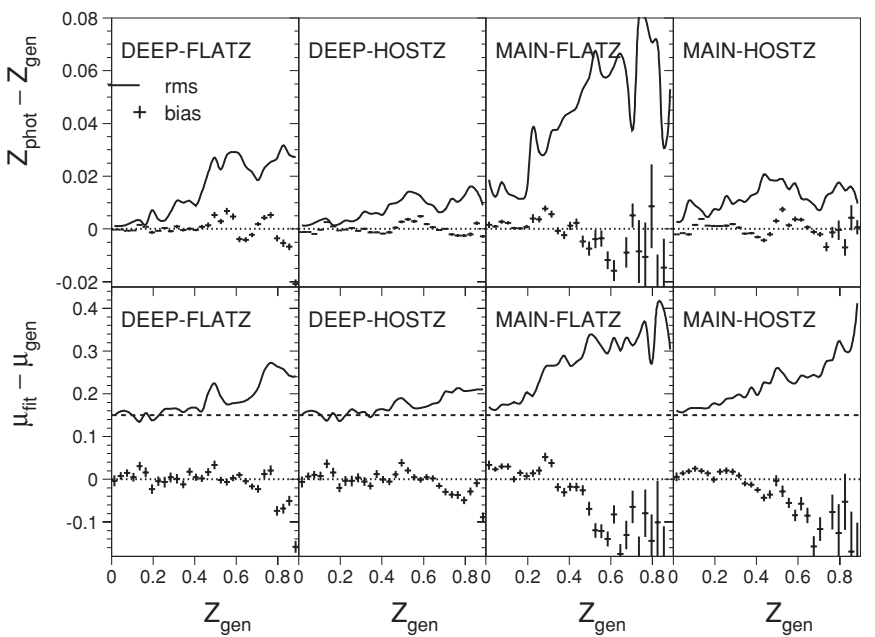

SALT2 Coherent mag-smearing in sim

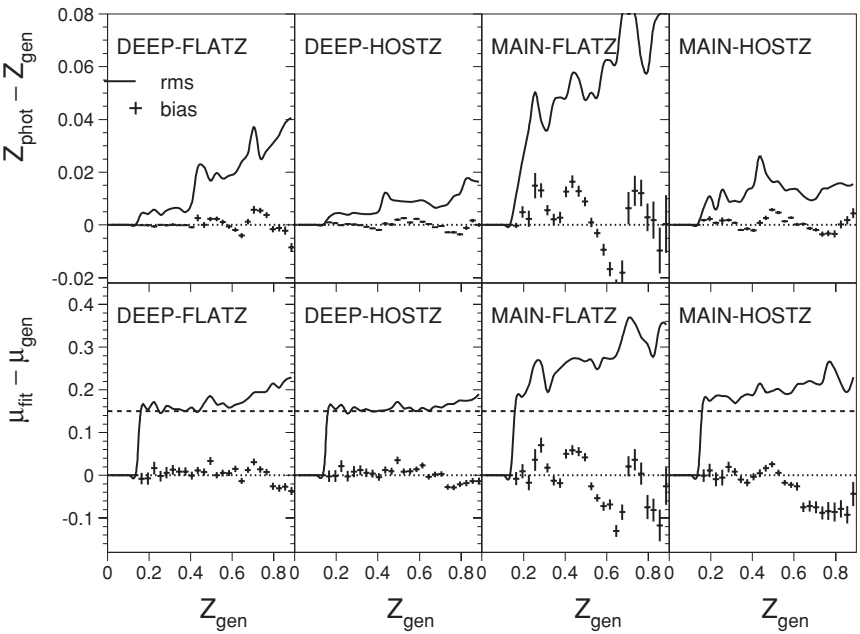

Figure 14. Bias (crosses) and rms (curve) on photo- $z$ residual $\left(z_{\text {phot }}-z_{\text {gen }}\right)$, and on distance modulus residual $\left(\mu_{\text {fit }}-\mu_{\text {gen }}\right)$ vs. the true redshift $\left(z_{\text {gen }}\right)$ in $z$-bins of width $\Delta Z=0.03$ for the LSST simulations. Each plot indicates DEEP or MAIN fields, and FLATZ (SN only) or HOSTZ (host-galaxy photo- $z$ prior). The simulation uses coherent $(0.15 \mathrm{mag})$ brightness fluctuations in all filters and is fitted with MLCS $2 \mathrm{~K} 2+\mathrm{Z}$ (left) and SALTII+Z (right). For $z<0.15$, the SALTII curves drop to zero because there are only two valid filters $(\mathrm{gr})$, and the data fail the three-filter requirement. The dashed horizontal lines in the $\mu_{\text {fit }}-\mu_{\text {gen }}$ plots show the rms contribution from intrinsic variations.
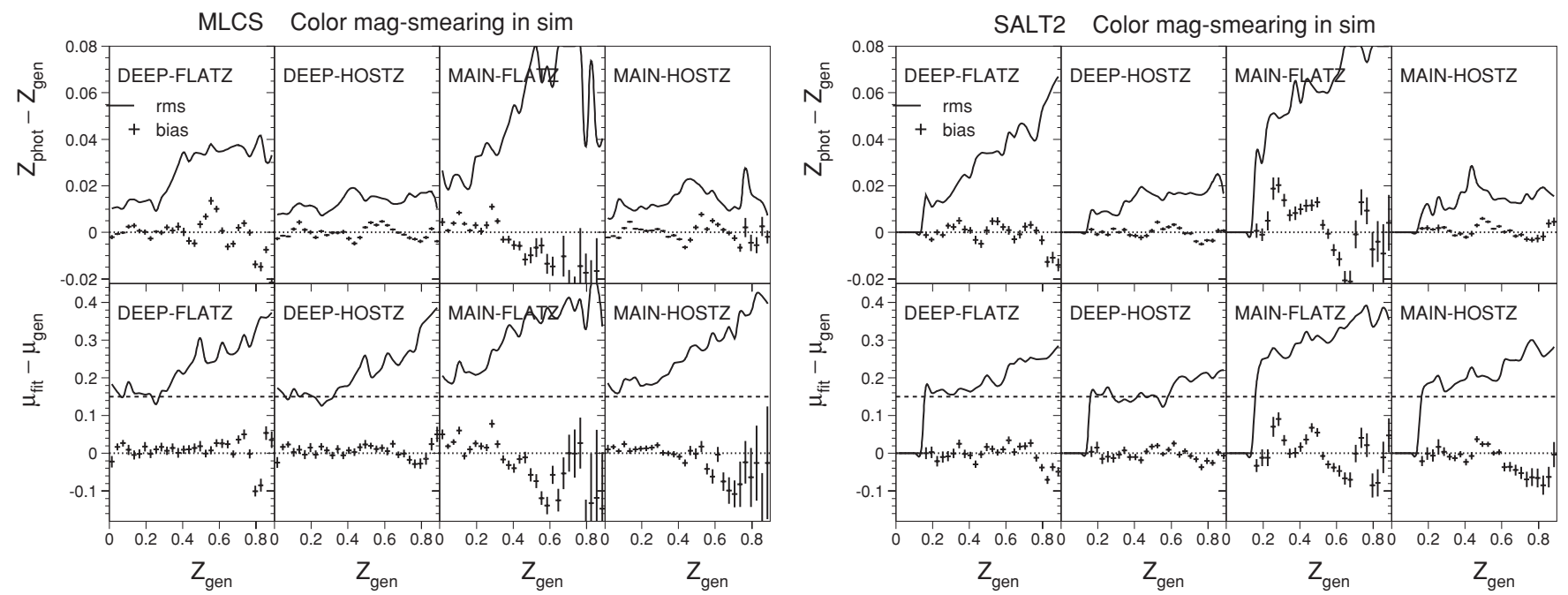

Figure 15. Same as Figure 14, except that the default color-smearing model is used in the simulation.

mag-smearing (Figure 13, left), $\eta_{0.15}=0$ in all cases. In this case, for the MAIN survey, $\eta_{0.10} \sim 0.01$ without a host-galaxy photo- $z$ prior, and $\eta_{0.10}$ is $\times 10$ smaller when the host-galaxy photo- $z$ prior is used. Using the default color-smearing model in the simulation (Figure 13, right), and fitting without a hostgalaxy photo- $z$ prior, $\eta_{0.15}<0.001$ for the DEEP survey, and it is somewhat larger for the MAIN survey: 0.004 and 0.012 using the MLCS $2 \mathrm{~K} 2+\mathrm{Z}$ and SALTII+Z methods, respectively. For $\eta_{0.10}$, the corresponding fractions are $\times 4$ larger. Using the SALTII $+Z$ method, the number of outliers is about $\times 3$ larger compared to MLCS2K2+Z; this difference could be related to the model, but it could also be an artifact of our implementation. We therefore make no claims that either method is more precise or has fewer catastrophic outliers. When the host-galaxy photo- $z$ prior is used, $\eta_{0.10}=\eta_{0.15}=0$ in all cases.

To quantify the precision of the LCFIT $+Z$ method, we have evaluated the bias and rms spread as a function of redshift for both the photo- $z$ and distance modulus $(\mu)$. Figure 14 shows the results based on simulations using the coherent mag-smearing model, and Figure 15 shows the results using the default color- smearing model. The main differences between using these two models of intrinsic variations are (1) the photo- $z$ rms goes to nearly zero at low redshift for the coherent mag-smearing model, but has a floor of about 0.01 for the color-smearing model and (2) the rms is slightly larger at high redshift for the color-smearing model.

Here we briefly summarize the precision based on simulations using the default color-smearing model and fitting without a host-galaxy photo- $z$ prior (see "FLATZ" panels in Figure 15). In the DEEP survey, the photo- $z$ rms precision is $\sim 0.01$ at low redshifts and rises to about 0.04 at the highest redshifts. The $\mathrm{RMS}_{\mu}$ precision is near the 0.15 mag floor at low redshifts and roughly doubles at the highest redshifts. In the MAIN survey, the photo- $z$ precision is about $\times 2$ worse compared to the DEEP field survey. The corresponding $\mathrm{RMS}_{\mu}$ precision is about $0.2 \mathrm{mag}$ at the lowest redshifts and also roughly doubles at the highest redshift. The photo- $z$ bias in the DEEP survey has wiggles of amplitude $\sim 0.01$ as a function of redshift. The $\mu$-bias wiggles are at most at the $0.01 \mathrm{mag}$ level, and are notably less apparent than those seen in the photo- $z$ bias. In the MAIN survey, the 
bias is noticeably larger and redshift dependent; the photo- $z$ bias reaches 0.02 and the $\mu$-bias reaches $0.1 \mathrm{mag}$. Both the photo- $z$ and $\mu$ biases are largest at $z_{\text {gen }} \sim 0.6$.

When fitting with a host-galaxy photo-z prior (see "HOSTZ" panels in Figure 15), the precision is significantly improved for both the photo- $z$ and $\mu$. In the MAIN survey, however, a redshift-dependent bias remains for $z_{\text {gen }}>0.6$.

Although the LSST photo- $z$ precision looks promising, we urge some caution in the interpretation of these simulations. If we consider the LSST DEEP-field subset over the same redshift range as the SDSS-II sample $(z<0.4)$, the forecast LSST photo$z$ precision (rms) is about $\times 3$ better than that for the SDSS-II data sample described in Section 5, reflecting the higher expected signal to noise and broader wavelength coverage. Although we are confident in extrapolating the rms precision based on the treatment of photon statistics and color smearing, we cannot rule out unknown systematic effects, primarily from the unknown source of intrinsic brightness variations, that could limit the photo- $z$ precision and accuracy. Concerning the photo- $z$ bias presented here, this should be considered a lower limit because the same light curve model has been used in both the simulation and in the LCFIT $+Z$ fit. The current MLCS2K2 and SALTII models have been shown to differ significantly in the ultraviolet region (K09), but such modeling errors have not been considered here. In future studies, fitting SALTII simulations with MLCS2K2 (and vice-versa) would likely give an upper limit on the photo-z bias, and may lead to additional clues about problems in the light curve models.

\section{COMPARISONS WITH COLOR-BASED REDSHIFT ESTIMATES}

Here we compare our LCFIT $+\mathrm{Z}$ results with color-based SN redshift estimates for the SNLS sample and for an MLCS2K2based simulation.

\subsection{Comparison with the SNLS Sample}

Wang (2007) determined color-based photometric redshifts for $40 \mathrm{SNe}$ Ia from the SNLS A06 sample. For the $20 \mathrm{SNe}$ used in the training, the author finds $\mathrm{RMS}_{\Delta_{z}}=0.03$; for the remaining $20 \mathrm{SNe}, \mathrm{RMS}_{\Delta_{z}}=0.05$. In our MLCS $2 \mathrm{~K} 2+\mathrm{Z}$ analysis, $37 \mathrm{SNe}$ satisfy the selection criteria, and $\mathrm{RMS}_{\Delta_{z}}=0.045$. For the SALTII+Z method, $37 \mathrm{SNe}$ satisfy the selection requirements, and $\mathrm{RMS}_{\Delta_{z}}=0.040$. By this metric, our LCFIT $+Z$ method works equally well compared to the color-based method. Since a list of SNe used by Wang (2007) is not available, we cannot make a more detailed comparison with the same subset.

\subsection{Comparison with MLCS2K2-based Simulations}

We compare photo- $z$ results of the two methods on simulations as described in Wang et al. (2007, hereafter WNW07). Following the procedure in WNW07, we simulate observer-frame filters $r i z$ using the MLCS2K2 model for redshifts $z<0.95$ (so that $r$-band data are always well defined within the model), fix the shape-luminosity parameter $\Delta=0$, generate a flat redshift distribution, and ignore intrinsic magnitude variations that introduce anomalous Hubble scatter. For each SN and each filter, the exposure time is adjusted so that $\mathrm{S} / \mathrm{N}=25$ at the epoch of peak brightness. In WNW07, only the peak fluxes are used and therefore the light curve sampling does not matter; to investigate the comparative effectiveness of our MLCs $2 \mathrm{~K} 2+\mathrm{Z}$ method, simulated light curves are sampled every 7 days in the observer frame.

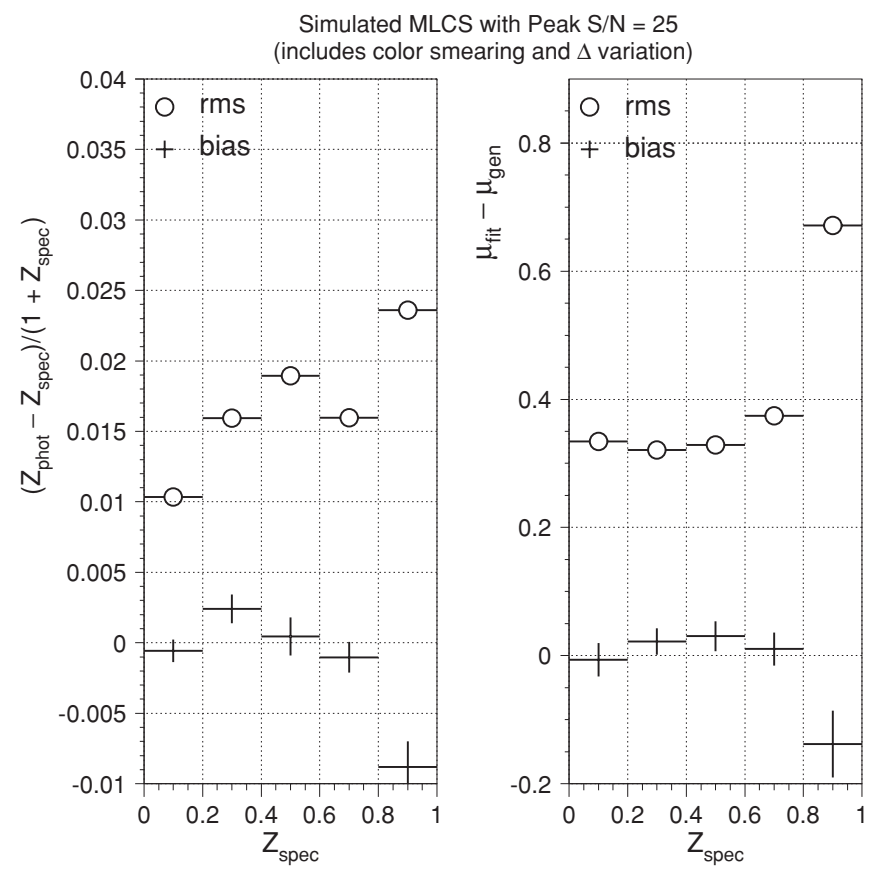

Figure 16. For MLCS $2 \mathrm{~K} 2$ simulations with peak $\mathrm{S} / \mathrm{N}=25$, fitted photo- $z$ bias and $\mathrm{RMS}_{\Delta_{z}}$ vs. $z_{\text {spec }}\left(\mathrm{left}\right.$ ), and distance modulus bias and $\mathrm{RMS}_{\mu}$ vs. $z_{\mathrm{spec}}$ (right). The simulations are generated according to the prescription of WNW07, but also including non-zero $\Delta$ and intrinsic color variations.

In the ideal case of no host-galaxy extinction $\left(A_{V}=0\right)$, WNW07 find RMS R $_{\Delta_{z}}=0.004$ with a mean bias of $5.4 \times$ $10^{-4}$; using MLCS $2 \mathrm{~K} 2+\mathrm{Z}$ under similar assumptions, we find $\mathrm{RMS}_{\Delta_{z}}=0.006$ and a mean bias of $(-1.7 \pm 2.1) \times 10^{-4}$. Including host-galaxy extinction in the simulation with a pdf $P\left(A_{V}\right)=\exp \left(-A_{V} / 0.46\right)$ and reddening parameter $R_{V}=3.1$, WNW07 find RMS ${ }_{\Delta_{z}}=0.044$ with a mean bias of 0.008 ; using MLCS $2 \mathrm{~K} 2+\mathrm{Z}$, under the same conditions we find $\mathrm{RMS}_{\Delta_{z}}=0.009$ and a mean bias of $(0.5 \pm 2.9) \times 10^{-4}$. The significantly improved precision with our MLCS $2 \mathrm{~K} 2+\mathrm{Z}$ method in these more realistic conditions is in part due to the increase in effective signal to noise that comes from using the entire light curve. In addition, shape and color information contained in the light curve enables the MLCs $2 \mathrm{~K} 2+\mathrm{Z}$ method to partially untangle color variations from extinction versus those produced by redshift.

Since the color-based method in WNV07 cannot untangle reddening from extinction and redshift, we have attempted a more fair comparison to the MLCS $2 \mathrm{~K} 2+\mathrm{Z}$ method in which $A_{V}$ is fixed to the mean generated extinction of $0.46 \mathrm{mag}$; in this case $\mathrm{RMS}_{\Delta_{z}}$ nearly doubles to 0.015 for the MLCS $2 \mathrm{~K} 2+\mathrm{Z}$ method, yet is nearly a factor of 3 smaller than the dispersion in WNV07. This difference is either due to the enhanced photon statistics from using the entire light curve in the MLCS $2 \mathrm{~K} 2+\mathrm{Z}$ method or from non-optimal training in the color-based method. To increase $\mathrm{RMS}_{\Delta_{z}}$ to the WNV07 value of 0.044 , the peak S/N must be reduced from 25 to less than 10 .

In the MLCS $2 \mathrm{~K} 2+\mathrm{Z}$ fits we have used $R_{V}=3.1$, the same value used in generating the simulation. Using the correct value for $R_{V}$ is an apparently unfair advantage over the WNW07 treatment, in which no assumptions are made about color variations. However, in practice the assumption about the value of $R_{V}$ makes little difference to the results: fitting with $R_{V}=2.2$ produces the same precision for $\Delta_{z}$, although the resulting distance moduli are biased by about 0.1 mag.

Even though the effects of host-galaxy extinction are included the simulation described in WNW07, this simulation is not 
Fit Correlations for MLCS Simulations with Peak $\mathrm{S} / \mathrm{N}=25$
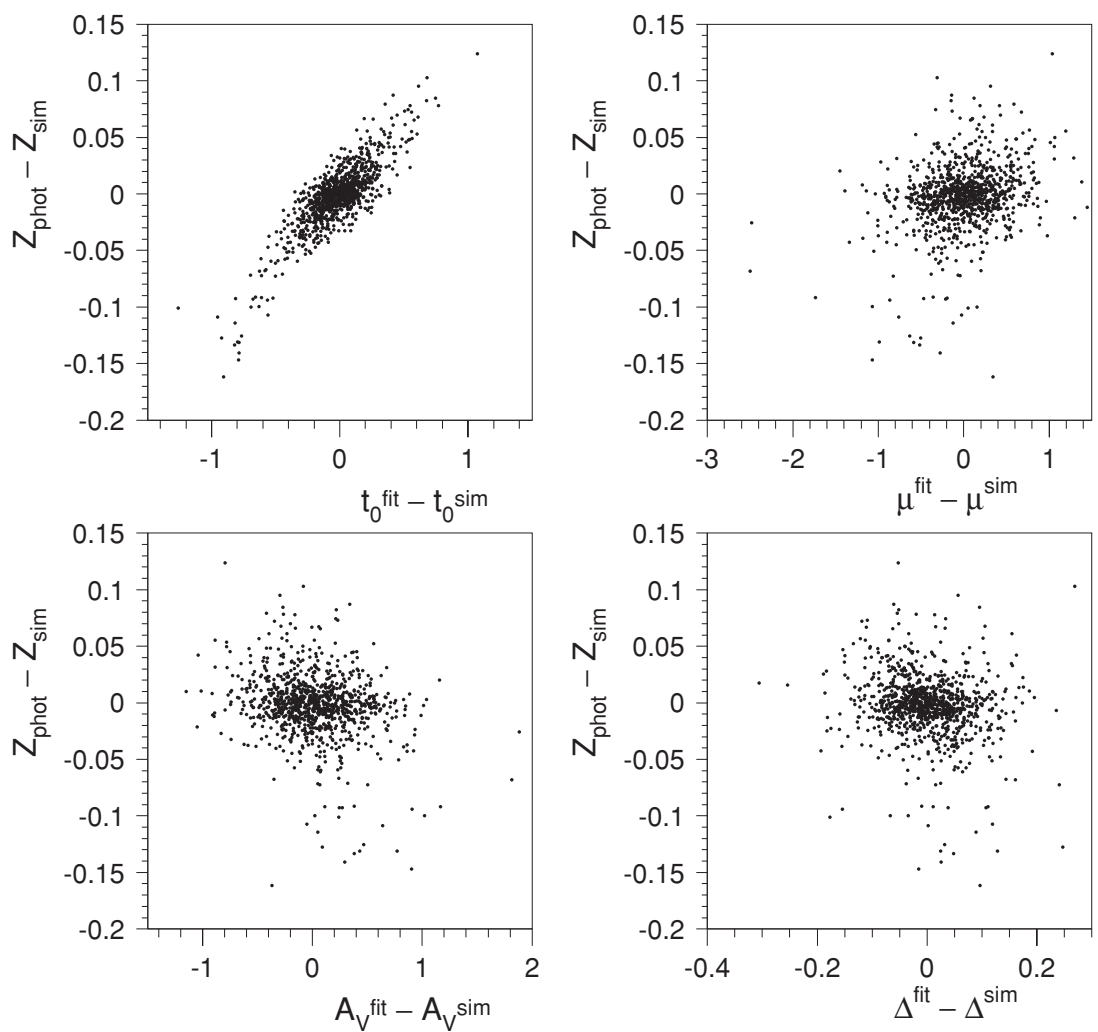

Figure 17. Fit-simulation photo- $z$ residual $\left(z_{\text {phot }}-z_{\text {gen }}\right)$ vs. fit-simulation residual for time of peak brightness $\left(t_{0}\right)$, distance modulus $(\mu)$, host-galaxy extinction $\left(A_{V}\right)$, and shape-luminosity parameter $(\Delta)$.

realistic because the shape-luminosity parameter $\Delta$ is fixed to zero, and there are no intrinsic magnitude variations. Simulating our best estimate for these effects (Section 3), the bias and scatter in the fitted photo- $z$ and distance modulus are shown as a function of $z_{\text {spec }}$ in Figure 16. For redshifts below 0.8 there is no significant bias in either the photo- $z$ or distance modulus. For $z>0.8$ the bias and scatter increase significantly. The nature of this high-redshift bias is illustrated in Figure 17, which shows the correlation between the fitted versus simulated photo$z$ difference and the fitted versus simulated difference for time of peak $\left(t_{0}\right)$, extinction, and shape-luminosity parameter. The photo- $z$ outliers are clearly correlated with $t_{0}$ outliers. Since the SN Ia color becomes redder with epoch, a misestimate of $t_{0}$ changes the apparent $\mathrm{SN}$ color, which is translated into an error in the redshift. The bias in $t_{0}$ is an artifact of the discrete MLCS2K2 passbands used to characterize the rest-frame light curves. For $\mathrm{SNe}$ simulated at redshifts $z>0.8$, the three observer-frame filters map into only two rest-frame filters: $r i z \rightarrow U B B$. In a small fraction of the light curve fits, however, the wrong filter-mapping $(r i z \rightarrow U B V)$ results in a smaller $\chi^{2}$. Since the $B$ - and $V$-band templates have different shapes, as well as a two-day shift in the time of peak brightness, the fitted $t_{0}$ is biased.

\section{CONCLUSIONS}

We have developed and tested a photometric redshift estimation method using SN Ia light curves within the framework of the MLCS2K2 and SALTII models and find that they result in similar photo- $z$ precision. We used an iterative fitting procedure to determine the valid observer-frame filters to use in the fits.
Applying this method to SDSS-II and SNLS data, we obtained an average photo- $z$ precision of $\mathrm{RMS}_{\Delta_{z}} \sim 0.04$. To reproduce a comparable level of precision in simulations, intrinsic color smearing is needed (Section 3) at the level of about 0.1 mag per passband or 0.14 mag per color. This empirical estimate of color smearing is consistent with but does not necessarily imply that there are random color variations in SN Ia light curves. However, this effect does indicate that there are additional sources of color variation that are not captured by the MLCS $2 \mathrm{~K} 2$ and SALTII light curve models.

We applied the LCFIT $+Z$ method to simulated LSST samples (Section 6). For the DEEP fields, the rms scatter of $z_{\text {phot }}-z_{\text {gen }}$ varies from 0.01 to 0.04 without using a host-galaxy photo- $z$ prior. For the MAIN survey, the photo- $z$ precision is about $\times 2$ worse. Using a host-galaxy photo- $z$ prior significantly reduces outliers and improves the overall precision. The next critical step is to apply this method to simulations that include non-Ia type $\mathrm{SNe}$ and estimate the resulting contamination of photometric $\mathrm{SN}$ Ia samples by core-collapse $\mathrm{SNe}$.

We gratefully acknowledge support from the Kavli Institute of Cosmological Physics at the University of Chicago, the National Science Foundation at Wayne State, and the Department of Energy at Fermilab, the University of Chicago, and Rutgers University. S.J. is grateful for the support of DOE grant DE-FG02-08ER41562. Funding for the creation and distribution of the SDSS and SDSS-II has been provided by the Alfred P. Sloan Foundation, the Participating Institutions, the National Science Foundation, the U.S. Department of Energy, the National Aeronautics and Space Administration, the Japanese Monbukagakusho, the Max Planck Society, and the 
Higher Education Funding Council for England. The SDSS Web site is http://www.sdss.org/.

The SDSS is managed by the Astrophysical Research Consortium for the Participating Institutions. The Participating Institutions are the American Museum of Natural History, Astrophysical Institute Potsdam, University of Basel, Cambridge University, Case Western Reserve University, University of Chicago, Drexel University, Fermilab, the Institute for Advanced Study, the Japan Participation Group, Johns Hopkins University, the Joint Institute for Nuclear Astrophysics, the Kavli Institute for Particle Astrophysics and Cosmology, the Korean Scientist Group, the Chinese Academy of Sciences (LAMOST), Los Alamos National Laboratory, the Max-Planck-Institute for Astronomy (MPA), the Max-Planck-Institute for Astrophysics (MPiA), New Mexico State University, Ohio State University, University of Pittsburgh, University of Portsmouth, Princeton University, the United States Naval Observatory, and the University of Washington.

This work is based in part on observations made at the following telescopes. The Hobby-Eberly Telescope (HET) is a joint project of the University of Texas at Austin, the Pennsylvania State University, Stanford University, Ludwig-MaximilliansUniversität München, and Georg-August-Universität Göttingen. The HET is named in honor of its principal benefactors, William P. Hobby and Robert E. Eberly. The Marcario Low-Resolution Spectrograph is named for Mike Marcario of High Lonesome Optics, who fabricated several optical elements for the instrument but died before its completion; it is a joint project of the Hobby-Eberly Telescope partnership and the Instituto de Astronomía de la Universidad Nacional Autónoma de México. The Apache Point Observatory $3.5 \mathrm{~m}$ telescope is owned and operated by the Astrophysical Research Consortium. We thank the observatory director, Suzanne Hawley, and site manager, Bruce Gillespie, for their support of this project. The Subaru Telescope is operated by the National Astronomical Observatory of Japan. The William Herschel Telescope is operated by the Isaac Newton Group on the island of La Palma in the Spanish Observatorio del Roque de los Muchachos of the Instituto de Astrofisica de Canarias. The W. M. Keck Observatory is operated as a scientific partnership among the California Institute of Technology, the University of California, and the National Aeronautics and Space Administration. The Observatory was made possible by the generous financial support of the W. M. Keck Foundation.

\section{APPENDIX A}

\section{FITTING ISSUES WITH UNKNOWN REDSHIFT}

Here we discuss our implementation of the five pre-fit issues mentioned in Section 4: (1) SN selection criteria that depend on knowing $T_{\text {rest }}=T_{\text {obs }} /(1+z)$ such as requiring measurements with a minimum and maximum $T_{\text {rest }}$ value; (2) determining which observer-frame filters (with mean wavelength $\bar{\lambda}_{\mathrm{f}}$ ) have $\bar{\lambda}_{\mathrm{f}} /\left(1+z_{\text {phot }}\right)$ within the valid wavelength range of the fitting model; (3) determining the valid rest-frame epoch range for the fitting model; (4) as $\bar{\lambda}_{\mathrm{f}} /\left(1+z_{\text {phot }}\right)$ maps into a different restframe filter (for MLCS2K2) there is a discontinuous change in the model error, and therefore the $\chi^{2}$ is not a continuous function of $z_{\text {phot }}$; and (5) determining robust initial fit parameter values.

The simplest way to handle $\mathrm{SN}$ selection criteria (issue 1) is to postpone such requirements until the fit has finished and then use the fitted photo- $z$ to determine the $T_{\text {rest }}$ values. This solution is not practical, however, because we often wish to remove poorly sampled light curves before fitting, thereby avoiding pathological fits that are of no interest. A safe way to apply $T_{\text {rest }}$-dependent requirements before fitting is to relax such cuts by a factor of $1+Z_{\max }$, where $Z_{\max }$ is a safe upper bound on all redshifts. For example, consider the requirements of a measurement with $T_{\text {rest }}<-6$ days and 21 days $<T_{\text {rest }}<$ 60 days. Using $Z_{\max }=0.5$ for the SDSS-II, the pre-fitted requirements are $T_{\text {rest }}<-4$ days and 14 days $<T_{\text {rest }}<90$ days. Any initial redshift value can be used to determine $T_{\text {rest }}$ as long as it is less than $Z_{\max }$. Although these $T_{\text {rest }}$-related requirements are relaxed, they are still useful for rejecting poorly sampled light curves; the nominal cut is applied after the fit using the fitted photo- $z$ value.

To determine the valid observer-frame filters (issue 2), the first-iteration photo- $z$ fit uses all filters, with a possible exception for those covering the ultraviolet region with a mean wavelength below about $4000 \AA$. The ultraviolet filter should be left out of the first iteration if it is rarely used, noting that it can be added back for the second fit iteration. The fundamental assumption about the fitting model is that extrapolating beyond the defined wavelength range gives reasonable magnitude estimates so that the fit converges and that the photo- $z$ bias is not too large. The (biased) photo- $z$ estimate is then used to determine which observer-frame filters to retain and to reject for the second fit iteration. Note that if an ultraviolet filter is excluded initially, then a low photo- $z$ value will result in the inclusion of this filter for the second iteration. If a filter is excluded, the $T_{\text {rest }}$-related requirements are re-tested; the fit stops if the light curve no longer has adequate sampling.

Since the first-iteration photo- $z\left(z_{\text {phot }}^{1}\right)$ is biased and has some uncertainty, a safety margin is used to determine which observerframe filters to keep. For each observer-frame filter (f) with mean wavelength $\bar{\lambda}_{\mathrm{f}}$, the valid redshift range for this filter is defined by

$$
\begin{aligned}
& z_{\min , \mathrm{f}}=\bar{\lambda}_{\mathrm{f}} / \lambda_{\max }^{\text {model }}-1, \\
& z_{\text {max }, \mathrm{f}}=\bar{\lambda}_{\mathrm{f}} / \lambda_{\min }^{\text {model }}-1,
\end{aligned}
$$

where $\lambda_{\min , \max }^{\bmod }$ are the minimum and maximum rest-frame wavelengths defined by the model. For example, the SALTII model is defined for rest-frame wavelengths $2900-7000 \AA$; the observerframe $i$ band $\left(\bar{\lambda}_{i}=7500 \AA\right)$ is therefore valid for redshifts above $z_{\min , i}=0.071$, and $g$ band $\left(\bar{\lambda}_{g}=4720 \AA\right)$ is valid for redshifts below $z_{\max , g}=0.63$. For this analysis, a filter is kept in the second iteration if the first-iteration fitted photo- $z$ satisfies

$$
z_{\mathrm{min}, \mathrm{f}}+\Delta z_{\text {phot }}^{\mathrm{cut}}<z_{\text {phot }}^{1}<z_{\mathrm{max}, \mathrm{f}}-\Delta z_{\text {phot }}^{\mathrm{cut}} .
$$

We have set $\Delta z_{\text {phot }}^{\text {cut }}=0.04$ and 0.03 for the SNLS and SDSS surveys, respectively. $\Delta z_{\text {phot }}^{\text {cut }}$ is smaller for the SDSS-II because the $\mathrm{S} / \mathrm{N}$ for $\mathrm{SNe}$ at $z_{\mathrm{min}, i}=0.07$ is larger than the $\mathrm{S} / \mathrm{N}$ for SNLS SNe at $z_{\max , g}=0.063$. The $\Delta z_{\text {phot }}^{\text {cut }}$ filter-selection cut has not been optimized; the optimal cut is likely to depend on the uncertainty of the host-galaxy photo- $z$ and may also depend on redshift.

This filter selection algorithm is illustrated in Figure 18. The solid histograms in the top two plots (SDSS data and simulation) show the true redshift $\left(z_{\mathrm{spec}}\right)$ distributions near $z_{\min , i}$ when the $i$ band is kept in the SALTII+Z fit. When the $i$ band is discarded (dashed), the entire light curve is discarded because of the requirement of having three filters. The gap between 

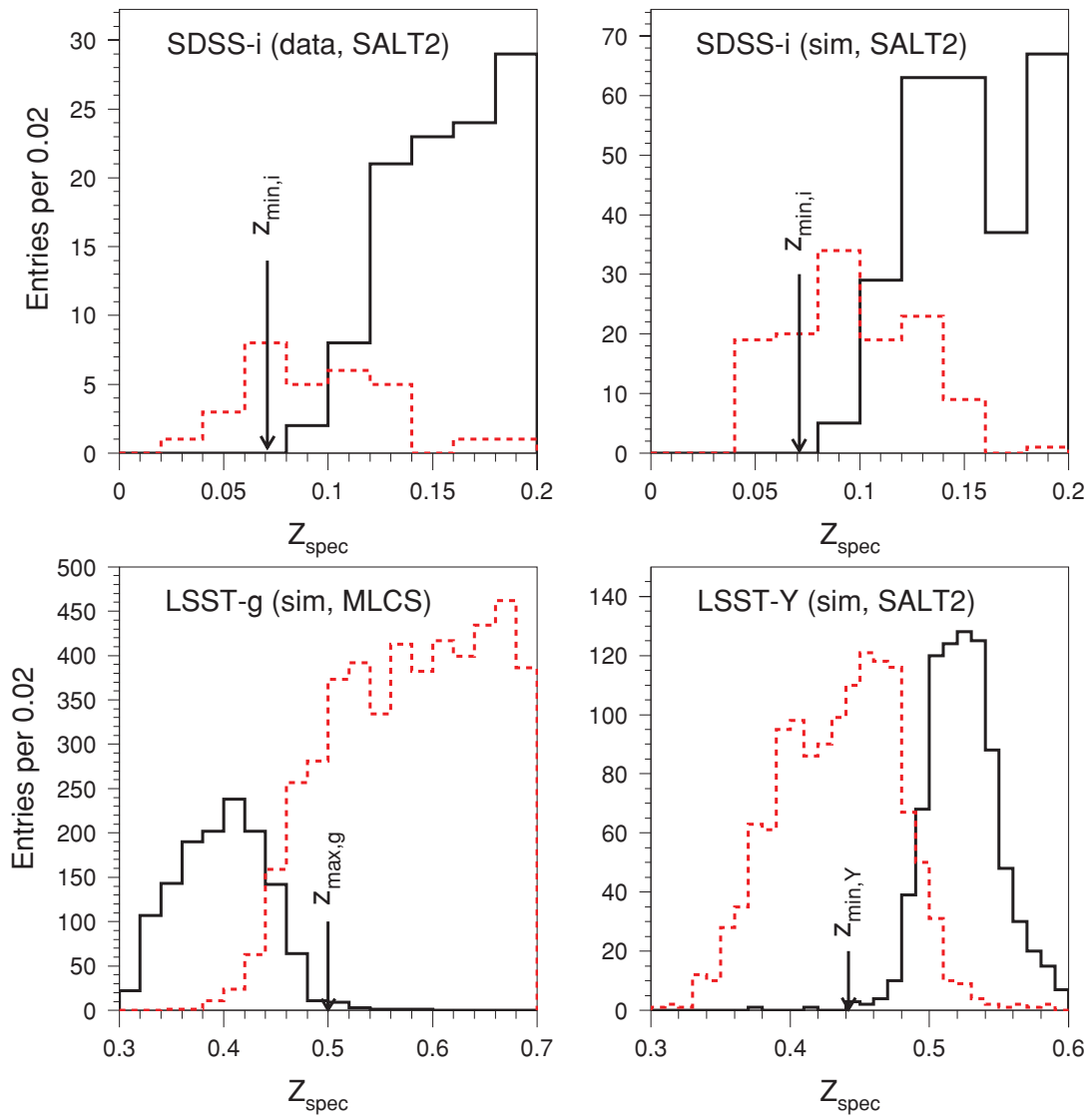

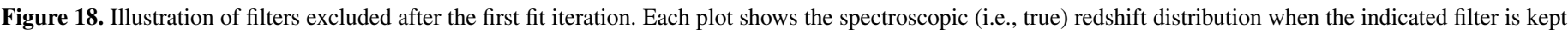

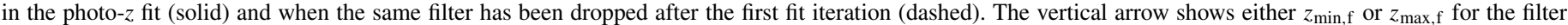
indicated. $z_{\text {spec }}$ is used only in making these plots, and is not used in the photo- $z$ fits.

(A color version of this figure is available in the online journal.)

$z_{\min , i}=0.071$ and the bulk distribution is due to the $\Delta z_{\text {phot }}^{\text {cut }}$ cut. The bottom two plots in Figure 18 illustrate cases for LSST when a dropped filter does not result in rejecting the entire light curve. When the LSST $g$ band is included in the MLCS $2 \mathrm{~K} 2+\mathrm{Z}$ fit (solid curve, lower left), the true redshift almost always satisfies the model-validity requirement $\left(z_{\mathrm{spec}}<0.5\right)$. The dashed curve shows that the $g$ band has been correctly excluded when the true redshift is above 0.5 , but we have also excluded this passband in some cases where it is valid (i.e., $z_{\text {spec }}<0.5$ ). The situation is similar for the LSST $Y$ band (lower right plot). For both passbands, a small number of light curve fits include these bands when they should have been excluded; potential biases from these invalid passbands should be accounted for in the assessment of systematic uncertainties on cosmological parameters.

The selection of rest-frame epochs (issue 3), which depends on $T_{\text {obs }} /\left(1+z_{\text {phot }}\right)$, is made in the same way as the selection of filters (issue 2). All measurements are included in the first fit iteration, and the initial photo- $z$ estimate is then used to select which epochs satisfy the epoch range of the model.

The $\chi^{2}$ continuity (issue 4 ) is an issue because the SNANA minimization is based on MINUIT, and this program relies on computing local derivatives with respect to the fitting parameters. This means that discontinuities in the $\chi^{2}$ function and its derivative can lead to fitting failures or pathological results. The problem can be solved by marginalizing, but the necessary five-dimensional integration requires $\sim 100$ times more CPU processing. In addition to the utility of using the much faster minimization program, it is useful to identify $\chi^{2}$ discontinuities because such functional pathologies usually correspond to unphysical behavior in the light curve model, and the model should be improved accordingly.

There are two main sources of $\chi^{2}$ discontinuity: (1) interpolating lookup tables and (2) filter-dependent model parameters. For class (1), appropriate numerical methods must be used to ensure continuity in both the function and its derivative. For class (2), the problem occurs for rest-frame models such as MLCS $2 \mathrm{~K} 2$, in which an infinitesimal change in $z_{\text {phot }}$ results in a different rest-frame filter for the model or a different color to use for spectral warping in the $K$-corrections. This step-function change in the model parameters results in a discontinuity in the model magnitude (or its error) as a function of $z_{\text {phot }}$. To prevent such sharp discontinuities, SNANA uses a smooth transition function $\left(A+B \tan ^{-1}\left(\lambda / \tau_{\lambda}\right)\right)$ to smoothly vary model parameters between neighboring filters.

Since the redshift and SN color are somewhat degenerate, initial parameter estimation (issue 5) is important so that MINUIT will find a global, rather than local, minimum. The initial color and photo- $z$ are determined from a crude $\chi^{2}$ minimization on a coarse grid: the color is varied in bins of 0.2 and the photo- $z$ is varied in bins of 0.04 . For each photo- $z$ value, an initial distance modulus estimate $\left(\mu_{\text {ini }}\right)$ is needed to ensure robust convergence of MINUIT. Although $\mu_{\text {ini }}$ is calculated from a standard $\Lambda$ CDM cosmology using a specific set of cosmological parameters, the fitted distance modulus is unconstrained and is therefore not biased by the $\mu_{\text {ini }}$ calculation. For SALTII, the initial $x_{0}$ value 
is estimated by inverting Equation (3). The shape-luminosity parameter is initialized to an average value: $\Delta=-0.1$ for MLCS $2 \mathrm{~K} 2$ and $x_{1}=0.0$ for SALTII. After the first fit iteration, the fitted parameters are used as initial values for the second fit iteration. When a filter is dropped and the first fit iteration is repeated, the fitted parameters are used as initial values, even though an invalid filter was used in the fit. If we do not use a coarse grid to initialize the color and photo- $z$ and simply start with average values, the photo- $z$ precision for the SDSSII sample is only slightly degraded. For the SNLS sample, however, which has a much larger redshift range compared to the SDSS-II, the photo- $z$ precision is degraded by a factor of 2 due to a significant number of catastrophic outliers.

\section{APPENDIX B}

\section{MARGINALIZATION}

Here we describe some of the details related to the marginalization. The minimized values and uncertainties are used to estimate the integration ranges: $\pm 4 \sigma$ around the minimized value for each parameter. The integration grid consists of $N_{\text {grid }}$ points per fit parameter or a total of $N_{\text {grid }}{ }^{5}$ integration cells. We find that $N_{\text {grid }}=11$ is a good compromise between precision and computing time. To improve calculation speed per integration cell, the $\chi^{2}$ calculation stops when the probability falls below $10^{-5}$.

The marginalization is repeated for either of the following cases: (1) the probability at any integration boundary is greater than 0.03 or (2) more than three one-dimensional bins (i.e., marginalized over the other four parameters) have a probability less than $10^{-4}$. In the first case the integration range is extended, while in the second case the integration range is reduced.

\section{REFERENCES}

Abdalla, F., et al. 2008, MNRAS, submitted (arXiv:0812.3831)

Astier, P., et al. 2006, A\&A, 447, 31

Benitez, N. 2000, ApJ, 536, 571

Bernstein, J. P., Kessler, R., Kuhlmann, S., \& Spinka, H. 2009, arXiv: 0906.2955

Dahlen, T., \& Goobar, A. 2002, PASP, 114, 284

Delgado, F., Cook, K., Miller, M., Allsman, R., \& Pierfederici, F. 2006, Proc. SPIE, 6270, 62701D

Frieman, J. A., et al. 2008, AJ, 135, 338

Fukugita, M., et al. 1996, AJ, 111, 1748

Gong, Y., Cooray, A., \& Chen, X. 2010, AJ, 709, 1420

Gunn, J. E., et al. 1998, AJ, 116, 3040

Gunn, J. E., et al. 2006, AJ, 131, 2332

Guy, J., et al. 2007, A\&A, 466, 11

Holtzman, J., et al. 2008, AJ, 136, 2306

Hsiao, E., et al. 2007, ApJ, 663, 1187

Ivezić, Ž., et al. 2008, arXiv:0805.2366

Jha, S., Riess, A. G., \& Kirshner, R. P. 2007, AJ, 659, 122

Kessler, R., et al. 2009a, ApJS, 185, 32

Kessler, R., et al. 2009b, PASP, 121, 1028

Kim, A., \& Miquel, R. 2007, Astropart. Phys., 28, 448

LSST Science Collaborations 2009, LSST Science Book, ver. 2.0, arXiv:0912.0201

Lupton, R., et al. 2001, in ASP Conf. Ser. 238, Astronomical Data Analysis Software and Systems X, ed. F. R. Harnden, Jr., F. A. Primini, \& H. E. Payne (San Francisco, CA: ASP), 269

Nobili, S., \& Goobar, A. 2008, A\&A, 487, 19

Nugent, P., et al. 2002, PASP, 114, 803

Palanque-Delabrouille, N., et al. 2009, arXiv:0911.1629

Sako, M., et al. 2008, AJ, 135, 348

Schlegel, D., et al. 2009, arXiv:0902.4680

Sullivan, M., et al. 2006, AJ, 131, 969

Wang, Y. 2007, ApJ, 654, L123

Wang, Y., Narayan, G., \& Wood-Vasey, M. 2007, MNRAS, 382, 377

York, D. G., et al. 2000, AJ, 120, 1579

Zheng, C., et al. 2008, AJ, 135, 1766 